\title{
Unconditional global well-posedness for the 3D Gross-Pitaevskii equation for data without finite energy
}

\author{
Hartmut Pecher
}

\begin{abstract}
The Cauchy problem for the Gross-Pitaevskii equation in three space dimensions is shown to have an unconditionally unique global solution for data of the form $1+H^{s}$ for $5 / 6<s<1$, which do not have necessarily finite energy. The proof uses the I-method which is complicated by the fact that no $L^{2}$-conservation law holds. This shows that earlier results of Bethuel-Saut for data of the form $1+H^{1}$ and Gérard for finite energy data remain true for this class of rough data.
\end{abstract}

Mathematics Subject Classification (2000). 35Q55, 35B60, 37L50.

Keywords. Gross-Pitaevskii equation, Global well-posedness, Fourier restriction norm method.

\section{Introduction and main results}

The Cauchy problem for the Gross-Pitaevskii equation in three space dimensions reads as follows

$$
\begin{aligned}
i \frac{\partial v}{\partial t}-\Delta v & =v\left(1-|v|^{2}\right) \\
v(x, 0) & =v_{0}(x)
\end{aligned}
$$

under the condition

$$
v \rightarrow 1 \text { as }|x| \rightarrow+\infty
$$

where $v: \mathbb{R}^{1+3} \rightarrow \mathbb{C}$.

This problem occurs in theoretical physics, e.g. Bose-Einstein condensation and superfluidity, see $[9,13,14]$.

Then one has the energy conservation law (see below)

$$
E(v(t))=\int\left(|\nabla v(x, t)|^{2}+\frac{1}{2}\left(|v(x, t)|^{2}-1\right)^{2}\right) d x=E\left(v_{0}\right) .
$$


Because the solution does not vanish at infinity the standard theory for nonlinear Schrödinger equations is not directly applicable and it is natural to consider instead $u=v-1$ for a solution $v$ of (1). Then $u$ satisfies the equivalent problem

$$
\begin{aligned}
i \frac{\partial u}{\partial t}-\Delta u+(1+u)\left(|u|^{2}+2 \operatorname{Re} u\right) & =0 \\
u(x, 0) & =u_{0}(x),
\end{aligned}
$$

under the condition

$$
u \rightarrow 0 \quad \text { as } \quad|x| \rightarrow+\infty .
$$

The real part of the $L^{2}$-scalar product of Eq. (5) with $\frac{\partial u}{\partial t}$ gives

$$
\frac{\partial}{\partial t} \int|\nabla u|^{2} d x+\frac{1}{2} \frac{\partial}{\partial t} \int\left(|u(t)|^{2}+2 \operatorname{Re} u(t)\right)^{2} d x=0,
$$

because

$$
\frac{1}{2} \frac{\partial}{\partial t}\left(\left(|u|^{2}+2 \operatorname{Re} u\right)^{2}\right)=2 \operatorname{Re}\left((1+u)\left(|u|^{2}+2 \operatorname{Re} u\right) \frac{\partial \bar{u}}{\partial t}\right) .
$$

This gives the energy conservation law

$$
E(u(t))=\int|\nabla u(t)|^{2} d x+\frac{1}{2} \int\left(|u(t)|^{2}+2 \operatorname{Re} u(t)\right)^{2} d x=E\left(u_{0}\right) .
$$

In terms of $v$ one gets (4). Remark that no conservation of $\|u(t)\|_{L^{2}}$ holds (in contrast to standard problems)!

We however get a bound for $\|u(t)\|_{L^{2}}=\|u(t)\|$ for finite energy data, which also belong to $L^{2}$, in the following way. The imaginary part of the scalar product of Eq. (5) with $u$ gives

$$
\frac{1}{2} \frac{\partial}{\partial t}\|u(t)\|^{2}-\int\left(|u|^{2}+2 \operatorname{Re} u\right) \operatorname{Im} u d x=0,
$$

because

$$
\operatorname{Im}(1+u)\left(|u|^{2}+2 \text { Re } u\right) \bar{u}=-\left(|u|^{2}+2 \text { Re } u\right) \operatorname{Im} u .
$$

This immediately implies

$$
\frac{\partial}{\partial t}\|u(t)\|^{2} \leq 2 \int\left(|u(t)|^{3}+2|u(t)|^{2}\right) d x
$$

We also get

$$
\frac{\partial}{\partial t}\|u(t)\|^{2} \leq 2\left(\int\left(|u(t)|^{2}+2 \operatorname{Re} u(t)\right)^{2} d x\right)^{\frac{1}{2}}\|u(t)\| \leq 2 \sqrt{2 E\left(u_{0}\right)}\|u(t)\|,
$$

which implies

$$
\|u(t)\|^{2} \leq\left\|u_{0}\right\|^{2}+\int_{0}^{t} 2 \sqrt{2 E\left(u_{0}\right)}\|u(s)\| d s,
$$

thus by a Gronwall type lemma

$$
\|u(t)\| \leq\left\|u_{0}\right\|+\sqrt{2 E\left(u_{0}\right)} t .
$$


For data $u_{0} \in H^{1}\left(\mathbb{R}^{3}\right)$ these considerations lead directly to an a-priori-bound of $\|\nabla u(t)\|_{L^{2}}^{2} \leq E(u(t))=E\left(u_{0}\right)$, which is finite, because $H^{1} \subset L^{4}$ by Sobolev's embedding theorem, and also to an a-priori bound of $\|u(t)\|_{L^{2}}$. Together with local well-posedness (cf. Theorem 2.1 below) this shows that our problem $(5),(6),(7)$ (and equivalently $(1),(2),(3)$ ) has a unique global solution $u \in C^{0}\left(\mathbb{R}, H^{1}\left(\mathbb{R}^{3}\right)\right)$.

The original proof was given by Bethuel and Saut [1], Appendix A. Later Gérard [7] proved global well-posedness in the larger energy space using Strichartz estimates in two and three space dimensions. Gallo [6] proved global well-posedness for more general nonlinearities for data with finite energy and space dimension $n \leq 4$.

In the work at hand we are now interested in global well-posedness for data without finite energy, more precisely we consider solutions $v=1+u$, where $u \in H^{s}\left(\mathbb{R}^{3}\right)$ for $s<1$. We apply the so called I-method introduced by Colliander, Keel, Staffilani, Takaoka and Tao [5] and successfully applied to various problems. There are two facts which complicate the problem: on one hand there is no scaling invariance and on the other hand no conservation law for the $L^{2}$-norm of $u$. As usual the energy conservation law is not directly applicable for $H^{s}$-data with $s<1$. However there is an "almost conservation law" for the modified energy $E(I u)$, which is well defined for $u \in H^{s}$ (see the definition of $I$ below). This leads to an a-priori bound of $\|\nabla I u(t)\|_{L^{2}}$, if $s$ is close enough to 1 , namely $s>5 / 6$. This can be shown to be enough for an a-priori bound also for $\|u(t)\|_{L^{2}}$, which together gives a bound for $\|u(t)\|_{H^{s}}$. A local wellposedness result in Bourgain type spaces $X^{s, \frac{1}{2}+}[0, T] \subset C^{0}\left([0, T], H^{s}\right)$ with existence time dependent only on $\left\|u_{0}\right\|_{H^{s}}$ completes the global well-posedness result in this space. We even get unconditional global well-posedness in the space $C^{0}\left([0, T], H^{s}\right)$ using a result of Kato [11]. This leads to the following main results (cf. the definition of the $X^{s, b}$-spaces below):

Theorem 1.1. Let $T>0, s>5 / 6$ and $u_{0} \in H^{s}\left(\mathbb{R}^{3}\right)$. The Cauchy problem (5),(6) has a unique global solution in $X^{s, \frac{1}{2}+}[0, T]$. This solution belongs to $C^{0}\left([0, T], H^{s}\left(\mathbb{R}^{3}\right)\right)$.

Combining this with the unconditional uniqueness result of T. Kato which we prove in Proposition 1.1 below we even get

Theorem 1.2. Let $T>0, s>5 / 6$ and $u_{0} \in H^{s}\left(\mathbb{R}^{3}\right)$. The Cauchy problem (5),6) has a unique global solution in $C^{0}\left([0, T], H^{s}\left(\mathbb{R}^{3}\right)\right)$. Equivalently the Cauchy problem (1),(2) has a unique global solution in $C^{0}\left([0, T], 1+H^{s}\left(\mathbb{R}^{3}\right)\right)$ for data $v_{0} \in 1+H^{s}\left(\mathbb{R}^{3}\right)$.

The following proposition for more general nonlinearities and arbitrary dimensions goes back to Kato [11]. We give the (short) proof in the special case of cubic polynomials as nonlinearity in three space dimensions.

Proposition 1.1. Assume $u_{0} \in H^{s}\left(\mathbb{R}^{3}\right)$. The Cauchy problem

$$
i \frac{\partial u}{\partial t}-\Delta u=F(u, \bar{u}), \quad u(0)=u_{0}
$$


where $F(u, \bar{u})$ is a polynomial of degree three, has at most one solution $u \in$ $C^{0}\left([0, T], H^{s}\left(\mathbb{R}^{3}\right)\right)$ for any $T>0$, provided $s \geq 2 / 3$.

Proof. Let $u, v \in C^{0}\left([0, T], H^{s}\left(\mathbb{R}^{3}\right)\right)$ be two solutions. By Sobolev's embedding $u, v \in C^{0}\left([0, T], L^{\frac{18}{5}}\right)$ using $s \geq 2 / 3$. By the Strichartz estimates (see below) for the inhomogeneous Schrödinger equation we get (ignoring complex conjugates, which play no role here)

$$
\begin{aligned}
\| u- & v\left\|_{L_{t}^{3} L_{x}^{\frac{18}{5}}}+\right\| u-v \|_{L_{t}^{\infty} L_{x}^{2}} \\
\lesssim & \left\|u^{3}-v^{3}\right\|_{L_{t}^{2} L_{x}^{\frac{6}{5}}}+\left\|u^{2}-v^{2}\right\|_{L_{t}^{2} L_{x}^{\frac{6}{5}}}+\|u-v\|_{L_{t}^{1} L_{x}^{2}} \\
\lesssim & \|u-v\|_{L_{t}^{3} L_{x}^{\frac{18}{5}}}\left(\|u\|_{L_{t}^{12} L_{x}^{\frac{18}{5}}}^{2}+\|v\|_{L_{t}^{12} L_{x}^{\frac{18}{5}}}^{2}\right) \\
& \quad+\|u-v\|_{L_{t}^{\infty} L_{x}^{2}}\left(\|u\|_{L_{t}^{2} L_{x}^{3}}+\|v\|_{L_{t}^{2} L_{x}^{3}}\right)+\|u-v\|_{L_{t}^{1} L_{x}^{2}} \\
\lesssim & \|u-v\|_{L_{t}^{3} L_{x}^{\frac{18}{5}}} T^{\frac{1}{6}}\left(\|u\|_{L_{t}^{\infty} H_{x}^{s}}^{2}+\|v\|_{L_{t}^{\infty} H_{x}^{s}}^{2}\right) \\
& \quad+\|u-v\|_{L_{t}^{\infty} L_{x}^{2}} T^{\frac{1}{2}}\left(\|u\|_{L_{t}^{\infty} H_{x}^{s}}+\|v\|_{L_{t}^{\infty} H_{x}^{s}}+1\right) \\
\lesssim & \frac{1}{2}\left(\|u-v\|_{L_{t}^{3} L_{x}^{\frac{18}{5}}}+\|u-v\|_{L_{t}^{\infty} L_{x}^{2}}\right),
\end{aligned}
$$

choosing $T$ small enough, which shows $u=v$.

We use the following notation and well-known facts: the multiplier $I=I_{N}$ is for given $s<1$ and $N \geq 1$ defined by

$$
\widehat{I_{N} f}(\xi):=m_{N}(\xi) \widehat{f}(\xi),
$$

where $\widehat{ }$ denotes the Fourier transform with respect to the space variables. Here $m_{N}(\xi)$ is a smooth, radially symmetric, nonincreasing function of $|\xi|$ with

$$
m_{N}(\xi)= \begin{cases}1 & |\xi| \leq N \\ \left(\frac{N}{|\xi|}\right)^{1-s} & |\xi| \geq 2 N\end{cases}
$$

We remark that $I: H^{s} \rightarrow H^{1}$ is a smoothing operator, so that especially $E(I u)$ is well-defined for $u \in H^{s}\left(\mathbb{R}^{3}\right)$ (remark that $H^{1}\left(\mathbb{R}^{3}\right) \subset L^{4}\left(\mathbb{R}^{3}\right)$ ).

We use the Bourgain type function space $X^{m, b}$ belonging to the Schrödinger equation $i u_{t}-\Delta u=0$, which is defined as follows: let ${ }^{\wedge}$ or $\mathcal{F}$ denote the Fourier transform with respect to space and time and $\mathcal{F}^{-1}$ its inverse. $X^{m, b}$ is the completion of $\mathcal{S}\left(\mathbb{R} \times \mathbb{R}^{3}\right)$ with respect to

$$
\|f\|_{X^{m, b}}=\left\|\langle\xi\rangle^{m}\langle\tau\rangle^{b} \mathcal{F}\left(e^{-i t \Delta} f(x, t)\right)\right\|_{L_{\xi, \tau}^{2}}=\left\|\langle\xi\rangle^{m}\left\langle\tau+|\xi|^{2}\right\rangle^{b} \widehat{f}(\xi, \tau)\right\|_{L_{\xi, \tau}^{2},},
$$

For a given time interval $I$ we define

$$
\|f\|_{X^{m, b}(I)}:=\inf _{g_{\mid I}=f}\|g\|_{X^{m, b}} .
$$

For $s \geq 0$ and $1 \leq r<\infty$ we denote by $H^{s, r}$ the standard Sobolev space, i.e. the completion of $C_{0}^{\infty}\left(\mathbb{R}^{3}\right)$ with respect to

$$
\|f\|_{H^{s, r}}=\left\|\mathcal{F}^{-1}\left(\langle\xi\rangle^{s} \widehat{f}(\xi)\right)\right\|_{L^{r}} .
$$


We recall the following facts about the solutions $u$ of the inhomogeneous linear Schrödinger equation (see e.g. [8])

$$
i u_{t}-\Delta u=F, \quad u(0)=f .
$$

For $b^{\prime}+1 \geq b \geq 0 \geq b^{\prime}>-1 / 2$ and $T \leq 1$ we have

$$
\|u\|_{X^{m, b}[0, T]} \lesssim\|f\|_{H^{m}}+T^{1+b^{\prime}-b}\|F\|_{X^{m, b^{\prime}}[0, T]} .
$$

For $1 / 2>b>b^{\prime} \geq 0$ or $0 \geq b>b^{\prime}>-1 / 2$ :

$$
\|f\|_{X^{m, b^{\prime}}[0, T]} \lesssim T^{b-b^{\prime}}\|f\|_{X^{m, b}[0, T]}
$$

(see e.g. [10], Lemma 1.10).

Fundamental are the following Strichartz type estimates for the solution $u$ of (9) in three space dimensions (see $[3,12])$ :

$$
\|u\|_{L^{q}\left(I, L^{r}\left(\mathbb{R}^{3}\right)\right)} \lesssim\|f\|_{\left.L^{2}\left(\mathbb{R}^{3}\right)\right)}+\|F\|_{L^{\tilde{q}^{\prime}}\left(I, L^{\tilde{r}^{\prime}}\left(\mathbb{R}^{3}\right)\right)}
$$

with implicit constant independent of the interval $I \subset \mathbb{R}$ for all pairs $(q, r),(\tilde{q}, \tilde{r})$ with $q, r, \tilde{q}, \tilde{r} \geq 2$ and $\frac{1}{q}+\frac{3}{2 r}=\frac{3}{4}, \frac{1}{\tilde{q}}+\frac{3}{2 \tilde{r}}=\frac{3}{4}$, where $\frac{1}{\tilde{q}}+\frac{1}{\tilde{q}^{\prime}}=1$ and $\frac{1}{\tilde{r}}+\frac{1}{\tilde{r}^{\prime}}=1$. This implies

$$
\|\psi\|_{L^{q}\left(I, L^{r}\left(\mathbb{R}^{3}\right)\right)} \lesssim\|\psi\|_{X^{0, \frac{1}{2}+}(I)} .
$$

For real numbers $a$ we denote by $a+, a++, a-$ and $a--$ the numbers $a+\epsilon, a+2 \epsilon, a-\epsilon$ and $a-2 \epsilon$, respectively, where $\epsilon>0$ is sufficiently small.

Of special interest is also a bilinear refinement, which goes back to Bourgain [2], namely the following frequency localized version in three dimensions:

Lemma 1.1. Let $u_{j}$ be given with supp $\widehat{u}_{j} \subset\left\{|\xi| \sim N_{j}\right\}(j=1,2), N_{1} \leq N_{2}$. Then the following estimates hold

$$
\begin{aligned}
&\left\|u_{1} u_{2}\right\|_{L_{x, t}^{2}} \lesssim \frac{N_{1}}{N_{2}^{\frac{1}{2}}}\left\|u_{1}\right\|_{X^{0, \frac{1}{2}}+}\left\|u_{2}\right\|_{X^{0, \frac{1}{2}+}}, \\
&\left\|u_{1} u_{2}\right\|_{L_{x, t}^{2}} \lesssim \frac{N_{1}^{1+}}{N_{2}^{\frac{1}{2}-}}\left\|u_{1}\right\|_{X^{0, \frac{1}{2}+}}\left\|u_{2}\right\|_{X^{0, \frac{1}{2}+}} \cdot
\end{aligned}
$$

Proof. For a proof of (10) we refer to Bourgain [2], Lemma 5 or Grünrock [10]. (11) follows by interpolation of (10) with the crude estimate

$$
\left\|u_{1} u_{2}\right\|_{L_{x t}^{2}} \lesssim\left\|u_{1}\right\|_{L_{t}^{\infty} L_{x}^{6+}}\left\|u_{2}\right\|_{L_{t}^{2} L_{x}^{3-}} \lesssim N_{1}^{1+}\left\|u_{1}\right\|_{X^{0, \frac{1}{2}+}}\left\|u_{2}\right\|_{X^{0, \frac{1}{2}+}},
$$

using $X^{0, \frac{1}{2}+} \subset L_{t}^{2} L_{x}^{3-}$ and Sobolev's embedding $\dot{H}^{1+} \subset L_{x}^{6+}$.

The paper is organized as follows: in Sect. 1 we prove two versions of a local well-posedness result for (5),(6), namely $u \in X^{s, \frac{1}{2}+}[0, \delta]$ for data $u_{0} \in H^{s}$ with $s>1 / 2$, and a modification where $\nabla I u \in X^{0, \frac{1}{2}+}[0, \delta]$ for data $\nabla I u_{0} \in L^{2}$, which is necessary in order to combine it with an almost conservation law for the modified energy $E(I u)$. In Sect. 2 we use these local results and bounds for the modified energy given in Sect. 3 in order to get the main theorem. It is namely shown that the bounds for the modified energy are enough to give also a uniform exponential bound for the $L^{2}$-norm of $u(t)$ and as a consequence for the $H^{s}$-norm for $u(t)$, which in view of the local well-posedness results suffices 
to get a global solution. In Sect. 3 we calculate $\frac{d}{d t} E(I u)$ for any solution of the equation $i \frac{\partial I u}{\partial t}-\Delta I u+I\left((1+u)\left(|u|^{2}+2 R e u\right)\right)=0$. The most complicated part is to estimate the time integrated terms which appear in $\frac{d}{d t} E(I u)$. Finally we show that these estimates control the modified energy $E(I u)$ uniformly on arbitrary time intervals $[0, T]$, provided $s>5 / 6$.

\section{Local well-posedness}

The following local well-posedness theorem is more or less standard.

Theorem 2.1. Assume $s>1 / 2$ and $u_{0} \in H^{s}\left(\mathbb{R}^{3}\right)$. Then the Cauchy problem (5),(6) is locally well-posed, i.e. there exists $T_{0}=T_{0}\left(\left\|u_{0}\right\|_{H^{s}}\right)$ such that there exists a unique solution $u \in X^{s, \frac{1}{2}+}\left(0, T_{0}\right)$. This solution belongs to $C^{0}\left(\left[0, T_{0}\right], H^{s}\left(\mathbb{R}^{3}\right)\right) . T_{0}$ can be chosen such that $T_{0} \sim \min \left(\left\|u_{0}\right\|_{H^{s}}^{-\frac{4}{2 s-1}-}, 1\right)$.

Proof. We have to estimate $\|F(u)\|_{X^{s,-\frac{1}{2}+}}$, where we define

$$
F(u)=(1+u)\left(|u|^{2}+2 \operatorname{Re} u\right) .
$$

We want to show

$$
\left\||u|^{2} u\right\|_{X^{s,-\frac{1}{2}++}} \lesssim T^{s-\frac{1}{2}-}\|u\|_{X^{s, \frac{1}{2}+}}^{3},
$$

where here and in the sequel we skip the interval $[0, T]$ in the $X^{s, b}[0, T]$ spaces. We ignore complex conjugates, because they play no role here, and use a fractional Leibniz rule and duality to reduce to the estimate

$$
\left\|u^{2}\langle D\rangle^{s} u \psi\right\|_{L_{x t}^{1}} \lesssim T^{s-\frac{1}{2}-}\|u\|_{X^{s, \frac{1}{2}+}}^{3}\|\psi\|_{X^{0, \frac{1}{2}--}} .
$$

We have

$$
\begin{aligned}
\left\|u^{2}\langle D\rangle^{s} u \psi\right\|_{L_{x t}^{1}} & \lesssim\left\|u^{2}\langle D\rangle^{s} u\right\|_{L_{t}^{1+} L_{x}^{2}}\|\psi\|_{L_{t}^{\infty-} L_{x}^{2}} \\
& \lesssim\|u\|_{L_{t}^{4+} L_{x}^{6}}^{2}\left\|\langle D\rangle^{s} u\right\|_{L_{t}^{2} L_{x}^{6}}\|\psi\|_{X^{0, \frac{1}{2}--}} \\
& \lesssim\|u\|_{L_{t}^{4+} H_{x}^{s, r}}^{2}\|u\|_{X^{s, \frac{1}{2}+}}\|\psi\|_{X^{0, \frac{1}{2}--}} \\
& \lesssim T^{s-\frac{1}{2}-}\|u\|_{L_{t}^{q} H_{x}^{s, r}}^{2}\|u\|_{X^{s, \frac{1}{2}+}}\|\psi\|_{X^{0, \frac{1}{2}--}} \\
& \lesssim T^{s-\frac{1}{2}-}\|u\|_{X^{s, \frac{1}{2}+}}^{3}\|\psi\|_{X^{0, \frac{1}{2}--}},
\end{aligned}
$$

where $\frac{1}{r}=\frac{1}{6}+\frac{s}{3}$, so that $H^{s, r} \subset L^{6}$, and $X^{0, \frac{1}{2}+} \subset L_{t}^{2} L_{x}^{6}$ by Strichartz, and $\frac{1}{q}=\frac{1-s}{2}$, so that $\frac{2}{q}=3\left(\frac{1}{2}-\frac{1}{r}\right)$, thus $X^{s, \frac{1}{2}+} \subset L_{t}^{q} H_{x}^{s, r}$ by Strichartz' estimate.

Similarly we get by Strichartz' estimate and Sobolev's embedding

$\left\|u\langle D\rangle^{s} u\right\|_{L_{t}^{1+} L_{x}^{2}} \lesssim T^{\frac{1}{2}-}\left\|u\langle D\rangle^{s} u\right\|_{L_{t}^{2} L_{x}^{2}} \lesssim T^{\frac{1}{2}-}\|u\|_{L_{t}^{\infty} L_{x}^{3}}\|u\|_{L_{t}^{2} H_{x}^{s, 6}} \lesssim T^{\frac{1}{2}-}\|u\|_{X^{s, \frac{1}{2}+}}^{2}$, thus

$$
\left\|u^{2}\right\|_{X^{s,-\frac{1}{2}++}} \lesssim T^{\frac{1}{2}-}\|u\|_{X^{s, \frac{1}{2}+}}^{2} .
$$

Finally

$$
\|u\|_{X^{s,-\frac{1}{2}++}} \lesssim T^{\frac{1}{2}-}\|u\|_{L_{t}^{2} H_{x}^{s}} \lesssim T^{1-}\|u\|_{X^{s, \frac{1}{2}+}} \cdot
$$


Similar estimates hold for the difference $\| F(u)-\left.F(v)\right|_{X^{0,-\frac{1}{2}+}}$. The standard Picard iteration shows the claimed result, where $T \leq 1$ has to be chosen that $T^{s-\frac{1}{2}-}\left\|u_{0}\right\|_{H^{s}}^{2} \lesssim 1$ and $T^{\frac{1}{2}-}\left\|u_{0}\right\|_{H^{s}} \lesssim 1$. Thus the choice as claimed in the theorem is possible.

Remarks: 1. A similar proof in spaces of the type $L_{t}^{p} L_{x}^{r}$ could also be given. This goes back to [4], where $s=1 / 2$ is included, but in this limiting case the existence time depends not only on $\left\|u_{0}\right\|_{H^{s}}$.

2. Theorem 2.1 shows that in order to get a global solution it is sufficient to have an a-priori bound of $\|u(t)\|_{H^{s}}$, if $s>1 / 2$.

We next prove a similar local well-posedness result involving the operator $I$.

Proposition 2.1. Assume $s>1 / 2$ and $\nabla I u_{0} \in L^{2}\left(\mathbb{R}^{3}\right)$. Then (after application of I) the problem (5),(6) has a unique local solution $u$ with $\nabla I u \in X^{0, \frac{1}{2}+}(0, \delta)$ and

$$
\|\nabla I u\|_{X^{0, \frac{1}{2}+}(0, \delta)} \leq \sqrt{2}\left\|\nabla I u_{0}\right\|_{L^{2}}
$$

where $\delta \leq 1$ can be chosen such that

$$
\left(\frac{\delta^{s-\frac{1}{2}-}}{N^{2(1-s)}}+\frac{\delta^{\frac{s}{2}-}}{N^{1-s}}+\delta^{\frac{1}{2}-}\right)\left\|\nabla I u_{0}\right\|_{L^{2}}^{2} \sim 1
$$

Proof. The cubic term in the nonlinearity will be estimated as follows:

$$
\left\|\nabla I\left(u_{1}, u_{2}, u_{3}\right)\right\|_{X^{0,-\frac{1}{2}++}} \lesssim\left(\frac{\delta^{s-\frac{1}{2}-}}{N^{2(1-s)}}+\frac{\delta^{\frac{s}{2}-}}{N^{1-s}}+\delta^{\frac{1}{2}-}\right) \prod_{i=1}^{3}\left\|\nabla I u_{i}\right\|_{X^{0, \frac{1}{2}+}}
$$

This follows from

$$
\begin{aligned}
A & :=\int_{0}^{\delta} \int_{*} M\left(\xi_{1}, \xi_{2}, \xi_{3}\right) \prod_{i=1}^{3} \widehat{u}_{i}\left(\xi_{i}, t\right) \widehat{\psi}\left(\xi_{4}, t\right) d \xi_{1} d \xi_{2} d \xi_{3} d \xi_{4} d t \\
& \lesssim\left(\frac{\delta^{s-\frac{1}{2}-}}{N^{2(1-s)}}+\frac{\delta^{\frac{s}{2}-}}{N^{1-s}}+\delta^{\frac{1}{2}-}\right) \prod_{i=1}^{3}\left\|u_{i}\right\|_{X^{0, \frac{1}{2}}+}\|\psi\|_{X^{0, \frac{1}{2}--}}
\end{aligned}
$$

where

$$
M\left(\xi_{1}, \xi_{2}, \xi_{3}\right):=\frac{m\left(\xi_{1}+\xi_{2}+\xi_{3}\right)}{m\left(\xi_{1}\right) m\left(\xi_{2}\right) m\left(\xi_{3}\right)} \frac{\left|\xi_{1}+\xi_{2}+\xi_{3}\right|}{\left|\xi_{1}\right|\left|\xi_{2}\right|\left|\xi_{3}\right|}
$$

and ${ }^{*}$ denotes integration over the region $\left\{\sum_{i=1}^{4} \xi_{i}=0\right\}$. We assume here and in the following w.l.o.g. that the Fourier transforms are nonnnegative. We also assume w.l.o.g. $\left|\xi_{1}\right| \geq\left|\xi_{2}\right| \geq\left|\xi_{3}\right|$. 
Case 1: $\left|\xi_{1}\right| \geq\left|\xi_{2}\right| \geq\left|\xi_{3}\right| \geq N$.

We first estimate the multiplier $M$. If $\left|\xi_{1}+\xi_{2}+\xi_{3}\right| \geq N$ we get

$$
\begin{aligned}
M\left(\xi_{1}, \xi_{2}, \xi_{3}\right) & \lesssim \prod_{i=1}^{3}\left(\frac{\left|\xi_{i}\right|}{N}\right)^{1-s} \frac{N^{1-s}}{\left|\xi_{1}+\xi_{2}+\xi_{3}\right|^{1-s}} \frac{\left|\xi_{1}+\xi_{2}+\xi_{3}\right|}{\left|\xi_{1}\right|\left|\xi_{2}\right|\left|\xi_{3}\right|} \\
& \lesssim \prod_{i=1}^{3}\left(\frac{\left|\xi_{i}\right|}{N}\right)^{1-s} \frac{N^{1-s}\left|\xi_{1}\right|^{s}}{\left|\xi_{1}\right|\left|\xi_{2}\right|\left|\xi_{3}\right|} \lesssim \frac{1}{\left|\xi_{2}\right|^{s}\left|\xi_{3}\right|^{s} N^{2(1-s)}},
\end{aligned}
$$

and if $\left|\xi_{1}+\xi_{2}+\xi_{3}\right| \leq N$ we have

$M\left(\xi_{1}, \xi_{2}, \xi_{3}\right) \lesssim \prod_{i=1}^{3}\left(\frac{\left|\xi_{i}\right|}{N}\right)^{1-s} \frac{N}{\left|\xi_{1}\right|\left|\xi_{2}\right|\left|\xi_{3}\right|} \lesssim \prod_{i=1}^{3} \frac{N^{s}}{\left|\xi_{i}\right|^{s} N^{2(1-s)}} \lesssim \frac{1}{\left|\xi_{2}\right|^{s}\left|\xi_{3}\right|^{s} N^{2(1-s)}}$

as before. This implies by Hölder's and Strichartz' inequality and Sobolev's embedding

$$
\begin{aligned}
A & \lesssim \frac{1}{N^{2(1-s)}}\|\psi\|_{L_{t}^{\tilde{q}} L_{x}^{2}}\left\|u_{1}\right\|_{L_{t}^{2} L_{x}^{6}}\left\|\mathcal{F}^{-1}\left(\frac{\widehat{u_{2}}}{\left|\xi_{2}\right|^{s}}\right)\right\|_{L_{t}^{q} L_{x}^{6}}\left\|\mathcal{F}^{-1}\left(\frac{\widehat{u_{3}}}{\left|\xi_{3}\right|^{s}}\right)\right\|_{L_{t}^{q} L_{x}^{6}} \\
& \lesssim \frac{\delta^{s-\frac{1}{2}-}}{N^{2(1-s)}}\|\psi\|_{L_{t}^{\infty-} L_{x}^{2}}\left\|u_{1}\right\|_{X^{0, \frac{1}{2}+}}\left\|u_{2}\right\|_{L_{t}^{q} L_{x}^{r}}\left\|u_{3}\right\|_{L_{t}^{q} L_{x}^{r}} \\
& \lesssim \frac{\delta^{s-\frac{1}{2}-}}{N^{2(1-s)}}\|\psi\|_{X^{0, \frac{1}{2}--}} \prod_{i=1}^{3}\left\|u_{i}\right\|_{X^{0, \frac{1}{2}+}} \cdot
\end{aligned}
$$

where $\frac{1}{q}=\frac{1}{2}-\frac{s}{2}, \frac{1}{\tilde{q}}=s-\frac{1}{2}$, such that $\dot{H}^{s, r} \subset L^{6}$ with $\frac{1}{r}=\frac{1}{6}+\frac{s}{3}$ and $\frac{1}{q}=\frac{3}{4}-\frac{3}{2 r}$.

Case 2: $\left|\xi_{1}\right| \geq\left|\xi_{2}\right| \geq N \geq\left|\xi_{3}\right|$.

The multiplier $M$ is estimated as follows: if $\left|\xi_{1}+\xi_{2}+\xi_{3}\right| \geq N$ we get

$$
\begin{aligned}
M\left(\xi_{1}, \xi_{2}, \xi_{3}\right) & \lesssim\left(\frac{\left|\xi_{1}\right|}{N}\right)^{1-s}\left(\frac{\left|\xi_{2}\right|}{N}\right)^{1-s} \frac{N^{1-s}}{\left|\xi_{1}+\xi_{2}+\xi_{3}\right|^{1-s}} \frac{\left|\xi_{1}+\xi_{2}+\xi_{3}\right|}{\left|\xi_{1}\right|\left|\xi_{2}\right|\left|\xi_{3}\right|} \\
& \lesssim \frac{1}{\left|\xi_{2}\right|^{s}\left|\xi_{3}\right| N^{1-s}},
\end{aligned}
$$

and if $\left|\xi_{1}+\xi_{2}+\xi_{3}\right| \leq N$ we also have

$$
M\left(\xi_{1}, \xi_{2}, \xi_{3}\right) \lesssim\left(\frac{\left|\xi_{1}\right|}{N}\right)^{1-s}\left(\frac{\left|\xi_{2}\right|}{N}\right)^{1-s} \frac{N}{\left|\xi_{1}\right|\left|\xi_{2}\right|\left|\xi_{3}\right|} \lesssim \frac{1}{\left|\xi_{2}\right|^{s}\left|\xi_{3}\right| N^{1-s}}
$$

This implies by Hölder, Strichartz and Sobolev

$$
\begin{aligned}
A & \lesssim \frac{1}{N^{1-s}}\|\psi\|_{L_{t}^{\frac{2}{s}} L_{x}^{2}}\left\|u_{1}\right\|_{L_{t}^{2} L_{x}^{6}}\left\|\mathcal{F}^{-1}\left(\left|\xi_{2}\right|^{-s} \widehat{u_{2}}\right)\right\|_{L_{t}^{q} L_{x}^{6}}\left\|\mathcal{F}^{-1}\left(\left|\xi_{3}\right|^{-1} \widehat{u_{3}}\right)\right\|_{L_{t}^{\infty} L_{x}^{6}} \\
& \lesssim \frac{1}{N^{1-s}} \delta^{\frac{s}{2}-}\|\psi\|_{L_{t}^{\infty-} L_{x}^{2}}\left\|u_{1}\right\| L_{L_{t}^{2} L_{x}^{6}}\left\|u_{2}\right\|_{L_{t}^{q} L_{x}^{r}}\left\|u_{3}\right\|_{L_{t}^{\infty} L_{x}^{2}} \\
& \lesssim \frac{1}{N^{1-s}} \delta^{\frac{s}{2}-}\|\psi\|_{X^{0, \frac{1}{2}--}} \prod_{i=1}^{3}\left\|u_{i}\right\|_{X^{0, \frac{1}{2}+}},
\end{aligned}
$$

where $\frac{1}{q}=\frac{1}{2}-\frac{s}{2}, \dot{H}_{x}^{s, r} \subset L_{x}^{6}$ for $\frac{1}{r}=\frac{1}{6}+\frac{s}{3}$, thus $\frac{1}{q}=\frac{3}{4}-\frac{3}{2 r}$ and $X^{0, \frac{1}{2}+} \subset L_{t}^{q} L_{x}^{r}$. 
Case 3: $\left|\xi_{1}\right| \geq N \geq\left|\xi_{2}\right| \geq\left|\xi_{3}\right|$ and $\left|\xi_{1}\right| \gg\left|\xi_{2}\right|$, or $N \geq\left|\xi_{1}\right| \geq\left|\xi_{2}\right| \geq\left|\xi_{3}\right|$. In these cases we have $M\left(\xi_{1}, \xi_{2}, \xi_{3}\right) \lesssim \frac{1}{\left|\xi_{2}\right|\left|\xi_{3}\right|}$, thus

$$
\begin{aligned}
A & \lesssim\|\psi\|_{L_{x t}^{2}}\left\|u_{1}\right\|_{L_{t}^{2} L_{x}^{6}}\left\|\mathcal{F}^{-1}\left(\left|\xi_{2}\right|^{-1} \widehat{u_{2}}\right)\right\|_{L_{t}^{\infty} L_{x}^{6}}\left\|\mathcal{F}^{-1}\left(\left|\xi_{3}\right|^{-1} \widehat{u_{3}}\right)\right\|_{L_{t}^{\infty} L_{x}^{6}} \\
& \lesssim \delta^{\frac{1}{2}-}\|\psi\|_{X^{0, \frac{1}{2}--}} \prod_{i=1}^{3}\left\|u_{i}\right\|_{X^{0, \frac{1}{2}+}}
\end{aligned}
$$

similarly as in case 2 . This implies (14).

Next we have to estimate the quadratic terms in the nonlinearity. We want to show

$$
\left\|\nabla I\left(u_{1} u_{2}\right)\right\|_{X^{0,-\frac{1}{2}++}} \lesssim \delta^{\frac{1}{2}-}\left\|\nabla I u_{1}\right\|_{X^{0, \frac{1}{2}+}}\left\|\nabla I u_{2}\right\|_{X^{0, \frac{1}{2}+}},
$$

which follows from

$$
\begin{aligned}
& B:=\int_{0}^{\delta} \int_{*} M\left(\xi_{1}, \xi_{2}\right) \widehat{u_{1}}\left(\xi_{1}, t\right) \widehat{u_{2}}\left(\xi_{1}, t\right) \widehat{\psi}\left(\xi_{3}, t\right) d \xi_{1} d \xi_{2} d \xi_{3} d t \\
& \lesssim \delta^{\frac{1}{2}-}\left\|u_{1}\right\|_{X^{0, \frac{1}{2}+}}\left\|u_{2}\right\|_{X^{0, \frac{1}{2}+}+\|\psi\|_{X^{0, \frac{1}{2}--}},}
\end{aligned}
$$

where $*$ denotes integration over the region $\left\{\xi_{1}+\xi_{2}+\xi_{3}=0\right\}$ and

$$
M\left(\xi_{1}, \xi_{2}\right):=\frac{m\left(\xi_{1}+\xi_{2}\right)}{m\left(\xi_{1}\right) m\left(\xi_{2}\right)} \frac{\left|\xi_{1}+\xi_{2}\right|}{\left|\xi_{1}\right|\left|\xi_{2}\right|} .
$$

Assuming w.l.o.g. $\left|\xi_{1}\right| \geq\left|\xi_{2}\right|$ we first consider

Case 1: $\left|\xi_{2}\right| \geq N$.

If $\left|\xi_{1}+\xi_{2}\right| \geq N$ we have

$$
M\left(\xi_{1}, \xi_{2}\right) \lesssim\left(\frac{\left|\xi_{1}\right|}{N}\right)^{1-s}\left(\frac{\left|\xi_{2}\right|}{N}\right)^{1-s} \frac{N^{1-s}}{\left|\xi_{1}+\xi_{2}\right|^{1-s}} \frac{\left|\xi_{1}+\xi_{2}\right|}{\left|\xi_{1}\right|\left|\xi_{2}\right|} \lesssim \frac{1}{\left|\xi_{2}\right|^{s} N^{1-s}}
$$

and in the case $\left|\xi_{1}+\xi_{2}\right| \leq N$ we also get

$$
M\left(\xi_{1}, \xi_{2}\right) \lesssim\left(\frac{\left|\xi_{1}\right|}{N}\right)^{1-s}\left(\frac{\left|\xi_{2}\right|}{N}\right)^{1-s} \frac{N}{\left|\xi_{1}\right|\left|\xi_{2}\right|} \lesssim \frac{1}{N^{2(1-s)}} \frac{N^{s} N^{1-s}}{\left|\xi_{2}\right|^{s}\left|\xi_{1}\right|^{s}} \lesssim \frac{1}{\left|\xi_{2}\right|^{s} N^{1-s}}
$$

so that by Strichartz' estimate using $s \geq \frac{1}{2}$ :

$$
\begin{aligned}
B & \lesssim \frac{1}{N^{1-s}}\|\psi\|_{L_{x t}^{2}}\left\|u_{1}\right\|_{L_{t}^{2} L_{x}^{6}}\left\|\mathcal{F}^{-1}\left(\frac{\widehat{u_{2}}}{\left|\xi_{2}\right|^{s}}\right)\right\|_{L_{t}^{\infty} L_{x}^{3}} \\
& \lesssim \frac{\delta^{\frac{1}{2}-}}{N^{1-s}}\|\psi\|_{X^{0, \frac{1}{2}--}}\left\|u_{1}\right\|_{X^{0, \frac{1}{2}+}}\left\|\mathcal{F}^{-1}\left(\frac{\widehat{u_{2}}}{\left|\xi_{2}\right|^{s}}\right)\right\|_{L_{t}^{\infty} \dot{H}_{x}^{\frac{1}{2}}} \\
& \lesssim \frac{\delta^{\frac{1}{2}-}}{N^{1-s}}\|\psi\|_{X^{0, \frac{1}{2}--}}\left\|u_{1}\right\|_{X^{0, \frac{1}{2}+}}\left\|u_{2}\right\|_{X^{0, \frac{1}{2}+}} \cdot
\end{aligned}
$$

Case 2: $N \geq\left|\xi_{2}\right|$.

If $\left|\xi_{1}\right| \gg\left|\xi_{2}\right|$ we have $M\left(\xi_{1}+\xi_{2}\right) \sim \frac{m\left(\xi_{1}+\xi_{2}\right)}{m\left(\xi_{1}\right) m\left(\xi_{2}\right)\left|\xi_{2}\right|} \sim \frac{1}{\left|\xi_{2}\right|}$, whereas, if $\left|\xi_{1}\right| \sim\left|\xi_{2}\right|$, we have $\left|\xi_{1}+\xi_{2}\right| \lesssim N$ and $m\left(\xi_{1}\right) \sim m\left(\xi_{2}\right) \sim m\left(\xi_{1}+\xi_{2}\right) \sim 1$, which leads to 
the same bound for $M\left(\xi_{1}, \xi_{2}\right)$. Thus

$$
\begin{aligned}
& B \lesssim\|\psi\|_{L_{x t}^{2}}\left\|u_{1}\right\|_{L_{t}^{2} L_{x}^{3}}\left\|\mathcal{F}^{-1}\left(\frac{\widehat{u_{2}}\left(\xi_{2}\right)}{\left|\xi_{2}\right|}\right)\right\|_{L_{t}^{\infty} L_{x}^{6}} \\
& \lesssim \delta^{\frac{1}{2}-}\|\psi\|_{X^{0, \frac{1}{2}--}}\left\|u_{1}\right\|_{X^{0, \frac{1}{2}+}}\left\|u_{2}\right\|_{X^{0, \frac{1}{2}+}} \cdot
\end{aligned}
$$

Finally

$$
\|\nabla I u\|_{X^{0,-\frac{1}{2}++}} \lesssim \delta^{\frac{1}{2}-}\|\nabla I u\|_{X^{0,0}} \lesssim \delta^{1-}\|\nabla I u\|_{X^{0, \frac{1}{2}+}} \cdot
$$

Similar estimates hold for the difference $\|\nabla I(F(u)-F(v))\|_{X^{0,-\frac{1}{2}+}}$.

A Picard iteration leads to the desired solution in $[0, \delta]$, where $\delta \leq 1$ has to be chosen such that

$$
\begin{aligned}
& \frac{\delta^{s-\frac{1}{2}-}}{N^{2(1-s)}}\left\|\nabla I u_{0}\right\|_{L^{2}}^{2} \lesssim 1, \quad \delta^{\frac{1}{2}-}\left\|\nabla I u_{0}\right\|_{L^{2}}^{2} \lesssim 1, \\
& \frac{\delta^{\frac{s}{2}-}}{N^{1-s}}\left\|\nabla I u_{0}\right\|_{L^{2}}^{2} \lesssim 1, \quad \delta^{\frac{1}{2}-}\left\|\nabla I u_{0}\right\|_{L^{2}} \lesssim 1 .
\end{aligned}
$$

Remark: We want to iterate this local existence theorem with time steps of equal length until we reach a given (large) time $T$. For this we need to control

$$
\|\nabla I u(t)\|_{L^{2}} \leq c(T) \quad \forall 0 \leq t \leq T .
$$

This is achieved for $u_{0} \in H^{s}$ and $s>5 / 6$ by giving uniform bounds of the modified energy $E(I u(t))$, which is done in Sect. 3 .

\section{Proof of Theorem 1.1}

Proof. Let us assume for the moment that (16) holds and show that this leads to the claim of Theorem 1.1. We thus have an a-priori bound for our local solution of Proposition 2.1 on any existence interval $[0, T]$, namely of

$$
\|\nabla I u(t)\|_{L^{2}} \sim\||\xi| \widehat{u}(\xi, t)\|_{L^{2}(\{|\xi| \leq N\})}+\left\||\xi|^{s} \widehat{u}(\xi, t)\right\|_{L^{2}(\{|\xi| \geq N\})} N^{1-s} .
$$

What remains to be given is an a-priori bound for $\|u(t)\|_{L^{2}}$ as a consequence of $(8)$.

Lemma 3.1. On any existence interval $[0, T]$ of our solution $u \in X^{s, \frac{1}{2}+}[0, T]$ we have $\|u(t)\|_{L^{2}\left(\mathbb{R}^{3}\right)} \leq c(T)$.

Proof. We smoothly decompose $\widehat{u}=\widehat{u_{1}}+\widehat{u_{2}}$ with supp $\widehat{u_{1}} \subset\{|\xi| \leq 2\}$ and supp $\widehat{u_{2}} \subset\{|\xi| \geq 1\}$. Then we have by Gagliardo-Nirenberg

$$
\begin{aligned}
\|u\|_{L^{3}} & \leq\left\|u_{1}\right\|_{L^{3}}+\left\|u_{2}\right\|_{L^{3}} \lesssim\left\|\nabla u_{1}\right\|_{L^{2}}^{\frac{1}{2}}\left\|u_{1}\right\|_{L^{2}}^{\frac{1}{2}}+\left\||D|^{\frac{1}{2}} u_{2}\right\|_{L^{2}} \\
& \lesssim\left\|\nabla u_{1}\right\|_{L^{2}}^{\frac{1}{2}}\left\|u_{1}\right\|_{L^{2}}^{\frac{1}{2}}+\left\||D|^{s} u_{2}\right\|_{L^{2}}^{\frac{1}{2 s}}\left\|u_{2}\right\|_{L^{2}}^{1-\frac{1}{2 s}} \\
& \lesssim\left\|\nabla u_{1}\right\|_{L^{2}}^{2}+\left\|u_{1}\right\|_{L^{2}}^{\frac{2}{3}}+\left\|u_{2}\right\|_{L^{2}}^{\frac{2}{3}}+\left\||D|^{s} u_{2}\right\|_{L^{2}}^{\frac{2}{3-2 s}}
\end{aligned}
$$


so that by (16) and (17) we get on $[0, T]$ :

$$
\begin{aligned}
\|u(t)\|_{L^{3}}^{3} & \lesssim\left\||\xi| \widehat{u_{1}}(\xi, t)\right\|_{L^{2}}^{6}+\left\|u_{1}(t)\right\|_{L^{2}}^{2}+\left\|u_{2}(t)\right\|_{L^{2}}^{2}+\left\||\xi|^{s} \widehat{u_{2}}(\xi, t)\right\|_{L^{2}}^{\frac{6}{3-2 s}} \\
& \leq c^{\prime}(T)\left(\|u(t)\|_{L^{2}}^{2}+1\right) .
\end{aligned}
$$

(8) gives

$$
\frac{d}{d t}\|u(t)\|_{L^{2}}^{2} \leq c^{\prime \prime}(T)\left(\|u(t)\|_{L^{2}}^{2}+1\right),
$$

so that Gronwall's lemma gives

$$
\|u(t)\|_{L^{2}}^{2}+1 \leq\left(\left\|u_{0}\right\|_{L^{2}}^{2}+1\right) e^{c^{\prime \prime}(T) T}
$$

on $[0, T]$.

Combining Lemma 3.1 with (16) and (17) we get an a-priori bound of $\|u(t)\|_{H^{s}}$. Together with Theorem 2.1 we immediately get Theorem 1.1.

\section{Estimates for the modified energy}

Application of the operator $I$ to Eq. (5) gives

$$
i \frac{\partial}{\partial t} I u-\Delta I u+I F(u)=0
$$

with

$$
F(u):=(1+u)\left(|u|^{2}+2 \operatorname{Re} u\right) .
$$

We define the modified energy

$$
E(I u)=\int|\nabla I u|^{2} d x+\frac{1}{2} \int\left(|I u|^{2}+2 \operatorname{Re} I u\right)^{2} d x .
$$

Of course one cannot expect that it is conserved, but we want to show an almost conservation law for it. We calculate its derivative as follows:

$$
\begin{aligned}
\frac{d}{d t} E(I u) & =2 \operatorname{Re}\left\langle-\Delta I u+\left(|I u|^{2}+2 \operatorname{Re} I u\right)(1+I u), I u_{t}\right\rangle \\
& =2 \operatorname{Re}\left\langle F(I u)-I F(u), I u_{t}\right\rangle
\end{aligned}
$$

by replacing $\Delta I u$ using (18). Next we replace $I u_{t}$ again by use of (18) and get

$$
\begin{aligned}
\frac{d}{d t} E(I u) & =\operatorname{Im}(\langle\nabla(F(I u)-I F(u)), \nabla I u\rangle+\langle F(I u)-I F(u), I F(u)\rangle) \\
& \leq|\langle\nabla(F(I u)-I F(u)), \nabla I u\rangle|+|\langle F(I u)-I F(u), I F(u)\rangle| .
\end{aligned}
$$

In order to control the increment of $E(I u)$ by (19) on the local existence interval $[0, \delta]$ we have to estimate several terms. We assume from now on $s \geq 3 / 4$.

1. Let us first consider the first term on the right hand side of (19). Here and in the following we ignore complex conjugates, because they are of no interest. We want to show

$$
\int_{0}^{\delta}\left|\left\langle\nabla\left(I\left(u^{3}\right)-(I u)^{3}\right), \nabla I u\right\rangle\right| d t \lesssim N^{-1+}\|\nabla I u\|_{X^{0, \frac{1}{2}+}}^{4} .
$$


This follows from

$$
A=\left|\int_{0}^{\delta} \int_{*} M\left(\xi_{1}, \xi_{2}, \xi_{3}\right) \prod_{i=1}^{4} \widehat{u_{i}}\left(\xi_{i}, t\right) d \xi_{1} d \xi_{2} d \xi_{3} d \xi_{4} d t\right| \lesssim N^{-1+} \prod_{i=1}^{4}\left\|u_{i}\right\|_{X^{0, \frac{1}{2}+}}
$$

where $^{*}$ denotes integration over the region $\left\{\sum_{i=1}^{4} \xi_{i}=0\right\}$ and

$$
M\left(\xi_{1}, \xi_{2}, \xi_{3}\right):=\frac{\left|m\left(\xi_{1}+\xi_{2}+\xi_{3}\right)-m\left(\xi_{1}\right) m\left(\xi_{2}\right) m\left(\xi_{3}\right)\right|}{m\left(\xi_{1}\right) m\left(\xi_{2}\right) m\left(\xi_{3}\right)} \frac{\left|\xi_{1}+\xi_{2}+\xi_{3}\right|}{\left|\xi_{1}\right|\left|\xi_{2}\right|\left|\xi_{3}\right|} .
$$

We assume here and in the following that the Fourier transforms are nonnegative w.l.o.g. In most of the cases we perform dyadic decompositions with respect to $\left|\xi_{i}\right|$, where $\left|\xi_{i}\right| \sim N_{i}$ with $N_{i}=2^{k_{i}}, k_{i} \in \mathbb{Z}$. In order to sum the dyadic parts at the end we always need a convergence generating factor $\frac{1 \wedge N_{\min }^{0+}}{N_{\max }^{0+}}$, where $N_{\min }$ and $N_{\max }$ is the smallest and the largest of the numbers $N_{i}$, respectively. $N_{\max } \geq N \geq 1$ can be assumed in all the cases, because otherwise our multiplier $M$ is identically zero.

In the term at hand we also assume w.l.o.g. $N_{1} \geq N_{2} \geq N_{3}$ and $N_{1} \geq N$.

Case 1: $N_{1} \geq N_{2} \geq N_{3} \gtrsim N \Rightarrow N_{4} \lesssim N_{1} \sim N_{\max }$.

We have for $s \geq 3 / 4$ :

$$
M\left(\xi_{1}, \xi_{2}, \xi_{3}\right) \lesssim\left(\frac{N_{1}}{N}\right)^{\frac{1}{4}}\left(\frac{N_{2}}{N}\right)^{\frac{1}{4}}\left(\frac{N_{3}}{N}\right)^{\frac{1}{4}} \frac{N_{1}}{N_{1} N_{2} N_{3}} .
$$

Thus by the bilinear Strichartz estimate (11) we get

$$
\begin{aligned}
& A \lesssim\left(\frac{N_{1}}{N}\right)^{\frac{1}{4}}\left(\frac{N_{2}}{N}\right)^{\frac{1}{4}}\left(\frac{N_{3}}{N}\right)^{\frac{1}{4}} \frac{1}{N_{2} N_{3}}\left\|u_{1} u_{3}\right\|_{L_{x t}^{2}}\left\|u_{2}\right\|_{L_{t}^{\infty} L_{x}^{3-}}\left\|u_{4}\right\|_{L_{t}^{2} L_{x}^{6+}} \\
& \lesssim\left(\frac{N_{1}}{N}\right)^{\frac{1}{4}}\left(\frac{N_{2}}{N}\right)^{\frac{1}{4}}\left(\frac{N_{3}}{N}\right)^{\frac{1}{4}} \frac{1}{N_{2} N_{3}} \frac{N_{3}^{1+}}{N_{1}^{\frac{1}{2}-}} N_{2}^{\frac{1}{2}-} N_{4}^{0+} \prod_{i=1}^{4}\left\|u_{i}\right\|_{X^{0, \frac{1}{2}+}} \\
& \lesssim \frac{N_{3}^{\frac{1}{4}+} N_{4}^{0+}}{N_{1}^{\frac{1}{4}-} N_{2}^{\frac{1}{4}+} N^{\frac{3}{4}}} \prod_{i=1}^{4}\left\|u_{i}\right\|_{X^{0, \frac{1}{2}+}} \\
& \lesssim \frac{1 \wedge N_{\min }^{0+}}{N_{\max }^{0+} N^{1-}} \prod_{i=1}^{4}\left\|u_{i}\right\|_{X^{0, \frac{1}{2}+}}
\end{aligned}
$$

Case 2: $N_{1} \geq N_{2} \gtrsim N \gtrsim N_{3}$.

This gives the same bound as in case 1 (without the factor $\left(\frac{N_{3}}{N}\right)^{\frac{1}{4}}$ ).

Case 3: $N_{1} \gtrsim N \gtrsim N_{2} \geq N_{3}$ and $N_{1} \gg N_{2}$.

By the mean value theorem we get

$$
M\left(\xi_{1}, \xi_{2}, \xi_{3}\right) \lesssim \frac{\left|\nabla m\left(\xi_{1}\right) \xi_{2}\right|}{m\left(\xi_{1}\right)} \frac{N_{1}}{N_{1} N_{2} N_{3}} \lesssim \frac{N_{2}}{N_{1}} \frac{N_{1}}{N_{1} N_{2} N_{3}}
$$


leading as in case 1 to the bound

$$
\begin{aligned}
A & \lesssim \frac{N_{2}}{N_{1}} \frac{N_{1}}{N_{1} N_{2} N_{3}} \frac{N_{3}^{1+} N_{2}^{\frac{1}{2}-} N_{4}^{0+}}{N_{1}^{\frac{1}{2}-}} \prod_{i=1}^{4}\left\|u_{i}\right\|_{X^{0, \frac{1}{2}+}} \\
& \lesssim \frac{N_{2}^{\frac{1}{2}-} N_{3}^{0+} N_{4}^{0+}}{N_{1}^{\frac{3}{2}-}} \prod_{i=1}^{4}\left\|u_{i}\right\|_{X^{0, \frac{1}{2}+}} \lesssim \frac{1 \wedge N_{\min }^{0+}}{N_{\max }^{0+} N^{1-}} \prod_{i=1}^{4}\left\|u_{i}\right\|_{X^{0, \frac{1}{2}+}} \cdot
\end{aligned}
$$

This proves (20) after dyadic summation over $N_{1}, N_{2}, N_{3}, N_{4}$.

2. We next want to show

$$
\int_{0}^{\delta}\left|\left\langle\nabla\left(I\left(u^{2}\right)-(I u)^{2}\right), \nabla I u\right\rangle\right| d t \lesssim N^{-1+} \delta^{\frac{1}{4}-}\|\nabla I u\|_{X^{0, \frac{1}{2}+}}^{3}
$$

which follows from

$$
B=\left|\int_{0}^{\delta} \int_{*} M\left(\xi_{1}, \xi_{2}, \xi_{3}\right) \prod_{i=1}^{3} \widehat{u}_{i}\left(\xi_{i}, t\right) d \xi_{1} d \xi_{2} d \xi_{3} d t\right| \lesssim N^{-1+} \delta^{\frac{1}{4}-} \prod_{i=1}^{3}\left\|u_{i}\right\|_{X^{0, \frac{1}{2}+}},
$$

where $^{*}$ denotes integration over the region $\left\{\sum_{i=1}^{3} \xi_{i}=0\right\}$ and

$$
M\left(\xi_{1}, \xi_{2}, \xi_{3}\right):=\frac{\left|m\left(\xi_{2}+\xi_{3}\right)-m\left(\xi_{2}\right) m\left(\xi_{3}\right)\right|}{m\left(\xi_{2}\right) m\left(\xi_{3}\right)} \frac{\left|\xi_{2}+\xi_{3}\right|}{\left|\xi_{2}\right|\left|\xi_{3}\right|} .
$$

Assume w.l.o.g. $\left|\xi_{2}\right| \geq\left|\xi_{3}\right|$ and $\left|\xi_{2}\right| \geq N \Rightarrow\left|\xi_{1}\right| \lesssim\left|\xi_{2}\right| \sim N_{\max }$.

Case 1: $N_{2} \sim N_{3} \gtrsim N$.

We have

$$
M\left(\xi_{1}, \xi_{2}, \xi_{3}\right) \lesssim \frac{1}{\left|m\left(\xi_{2}\right)\right|^{2} N_{2}} \lesssim\left(\frac{N_{2}}{N}\right)^{\frac{1}{2}} \frac{1}{N_{2}}
$$

Using $\dot{H}_{x}^{0+, 3} \subset L_{x}^{3+}$ and $X^{0, \frac{1}{2}+} \subset L_{t}^{4+} L_{x}^{3-}$ we get

$$
\begin{aligned}
B & \lesssim\left(\frac{N_{2}}{N}\right)^{\frac{1}{2}} \frac{1}{N_{2}}\left\|u_{1}\right\|_{L_{x t}^{3+}}\left\|u_{2}\right\|_{L_{x t}^{3-}}\left\|u_{3}\right\|_{L_{x t}^{3+}} \lesssim \frac{\delta^{\frac{1}{4}-} N_{1}^{0+} N_{3}^{0+}}{N_{\max }^{0+} N^{1-}} \prod_{i=1}^{3}\left\|u_{i}\right\|_{L_{t}^{4+} L_{x}^{3-}} \\
& \lesssim \frac{\delta^{\frac{1}{4}-}\left(1 \wedge N_{\min }^{0+}\right)}{N_{\max }^{0+} N^{1-}} \prod_{i=1}^{3}\left\|u_{i}\right\|_{X^{0, \frac{1}{2}+}} \cdot
\end{aligned}
$$

Case 2: $N_{1} \sim N_{3} \Rightarrow N_{2} \lesssim N_{1}$.

Because (see above) $N_{1} \lesssim N_{2}$ we have $N_{1} \sim N_{2} \sim N_{3}$, which was already considered in case 1 .

Case 3: $\left|\xi_{1}\right| \sim\left|\xi_{2}\right| \Rightarrow\left|\xi_{3}\right| \lesssim\left|\xi_{1}\right|$ and $\left|\xi_{3}\right| \ll\left|\xi_{1}\right| \sim\left|\xi_{2}\right|$.

a. If $\left|\xi_{3}\right| \gtrsim N$, we get as in case 1

$$
M\left(\xi_{1}, \xi_{2}, \xi_{3}\right) \lesssim \frac{1}{m\left(\xi_{3}\right)\left|\xi_{3}\right|} \lesssim\left(\frac{\left|\xi_{3}\right|}{N}\right)^{\frac{1}{4}} \frac{1}{\left|\xi_{3}\right|} \lesssim \frac{1}{N}
$$

Thus by Strichartz' estimate

$$
B \lesssim \frac{1}{N} \prod_{i=1}^{3}\left\|u_{i}\right\|_{L_{x t}^{3}} \lesssim \frac{\delta^{\frac{1}{4}}}{N} \prod_{i=1}^{3}\left\|u_{i}\right\|_{L_{t}^{4} L_{x}^{3}} \lesssim \frac{\delta^{\frac{1}{4}}}{N} \prod_{i=1}^{3}\left\|u_{i}\right\|_{X^{0, \frac{1}{2}+}} .
$$


b. If $\left|\xi_{3}\right| \lesssim N$ we estimate

$$
M\left(\xi_{1}, \xi_{2}, \xi_{3}\right) \sim \frac{\left|(\nabla m)\left(\xi_{2}\right) \xi_{3}\right|}{m\left(\xi_{2}\right)\left|\xi_{3}\right|} \sim \frac{1}{\left|\xi_{2}\right|} \lesssim \frac{1}{N}
$$

leading to the same bound as in a.

This proves (21).

3. Next we consider the second term on the right hand side of (19) and want to show

$$
\int_{0}^{\delta}\left|\left\langle I\left(u^{3}\right)-(I u)^{3}, I\left(u^{3}\right)\right\rangle\right| d t \lesssim N^{-2+}\|\nabla I u\|_{X^{0, \frac{1}{2}+}}^{6} .
$$

This means that we have to show

$$
C=\left|\int_{0}^{\delta} \int_{*} M\left(\xi_{1}, \ldots, \xi_{6}\right) \prod_{i=1}^{6} \widehat{u_{i}}\left(\xi_{i}, t\right) d \xi_{1} \ldots d \xi_{6} d t\right| \lesssim N^{-2+} \prod_{i=1}^{6}\left\|u_{i}\right\|_{X^{0, \frac{1}{2}+}},
$$

where

$$
\begin{aligned}
& M\left(\xi_{1}, \ldots, \xi_{6}\right):=\frac{\left|m\left(\xi_{1}+\xi_{2}+\xi_{3}\right)-m\left(\xi_{1}\right) m\left(\xi_{2}\right) m\left(\xi_{3}\right)\right|}{m\left(\xi_{1}\right) m\left(\xi_{2}\right) m\left(\xi_{3}\right)} \\
& \quad \times \frac{m\left(\xi_{4}+\xi_{5}+\xi_{6}\right)}{m\left(\xi_{4}\right) m\left(\xi_{5}\right) m\left(\xi_{6}\right)} \prod_{i=1}^{6}\left|\xi_{i}\right|^{-1} .
\end{aligned}
$$

Assume w.l.o.g. $N_{1} \geq N_{2} \geq N_{3}, N_{1} \geq N$ and $N_{4} \geq N_{5} \geq N_{6}$.

Case 1: $N_{4} \geq N_{5} \geq N_{6} \geq N$.

a. $N_{1} \geq N_{2} \geq N_{3} \geq N$.

We have

$$
M\left(\xi_{1}, \ldots, \xi_{6}\right) \lesssim \prod_{i=1}^{6}\left(\frac{N_{i}}{N}\right)^{\frac{1}{4}} \prod_{i=1}^{6} N_{i}^{-1} .
$$

Thus by Strichartz and Sobolev:

$$
\begin{aligned}
C & \lesssim \prod_{i=1}^{6}\left(\frac{N_{i}}{N}\right)^{\frac{1}{4}} \prod_{i=1}^{6} N_{i}^{-1}\left\|u_{1}\right\|_{L_{t}^{2} L_{x}^{6}}\left\|u_{2}\right\|_{L_{t}^{\infty} L_{x}^{3}}\left\|u_{4}\right\|_{L_{t}^{\infty} L_{x}^{3}}\left\|u_{5}\right\|_{L_{t}^{2} L_{x}^{6}}\left\|u_{3}\right\|_{L_{x t}^{\infty}}\left\|u_{6}\right\|_{L_{x t}^{\infty}} \\
& \lesssim \frac{1}{\prod_{i=1}^{6} N_{i}^{\frac{3}{4}} N^{\frac{3}{2}}} N_{2}^{\frac{1}{2}} N_{4}^{\frac{1}{2}} N_{3}^{\frac{3}{2}+} N_{6}^{\frac{3}{2}+} \prod_{i=1}^{6}\left\|u_{i}\right\|_{X^{0, \frac{1}{2}+}} \\
& \lesssim \frac{N_{3}^{\frac{3}{4}} N_{6}^{\frac{3}{4}+}}{N_{1}^{\frac{3}{4}} N_{2}^{\frac{1}{4}} N_{4}^{\frac{1}{4}} N_{5}^{\frac{3}{4}} N^{\frac{3}{2}}} \prod_{i=1}^{6}\left\|u_{i}\right\|_{X^{0, \frac{1}{2}+}} \\
& \lesssim \frac{1 \wedge N_{\min }^{0+}}{N_{1}^{0+} N_{2}^{\frac{1}{4}-} N_{4}^{0+} N_{5}^{\frac{1}{4}-} N^{\frac{3}{2}}} \prod_{i=1}^{6}\left\|u_{i}\right\|_{X^{0, \frac{1}{2}+}} \\
& \lesssim \frac{1 \wedge N_{\min }^{0+} \prod^{6}\left\|u_{i}\right\|_{X^{0, \frac{1}{2}+}} \cdot}{N_{\max }^{0+} N^{2-}} \prod_{i=1}
\end{aligned}
$$

b. $N_{1} \geq N_{2} \geq N \geq N_{3}$. 
This can be handled similarly as case a. without the factor $\left(\frac{N_{3}}{N}\right)^{\frac{1}{4}}$. Thus

$$
\begin{aligned}
C & \lesssim \frac{N_{3}^{\frac{1}{2}+} N_{6}^{\frac{3}{4}+}}{N_{1}^{\frac{3}{4}} N_{2}^{\frac{1}{4}} N_{4}^{\frac{1}{4}} N_{5}^{\frac{3}{4}} N^{\frac{5}{4}}} \prod_{i=1}^{6}\left\|u_{i}\right\|_{X^{0, \frac{1}{2}+}} \lesssim \frac{1 \wedge N_{\min }^{0+}}{N_{1}^{0+} N_{2}^{\frac{1}{2}-} N_{4}^{0+} N_{5}^{\frac{1}{4}-} N^{\frac{5}{4}}} \prod_{i=1}^{6}\left\|u_{i}\right\|_{X^{0, \frac{1}{2}+}} \\
& \lesssim \frac{1 \wedge N_{\min }^{0+}}{N_{\max }^{0+} N^{2-}} \prod_{i=1}^{6}\left\|u_{i}\right\|_{X^{0, \frac{1}{2}+}} \cdot
\end{aligned}
$$

c. $N_{1} \geq N \geq N_{2} \geq N_{3}$.

This can be handled as case b. without the factor $\left(\frac{N_{2}}{N}\right)^{\frac{1}{4}}$ leading to the bound

$$
\begin{aligned}
C & \lesssim \frac{N_{3}^{\frac{1}{2}+} N_{6}^{\frac{3}{4}+}}{N_{1}^{\frac{3}{4}} N_{2}^{\frac{1}{2}} N_{4}^{\frac{1}{4}} N_{5}^{\frac{3}{4}} N} \prod_{i=1}^{6}\left\|u_{i}\right\|_{X^{0, \frac{1}{2}+}} \lesssim \frac{1 \wedge N_{\min }^{0+}}{N_{1}^{\frac{3}{4}-} N_{4}^{\frac{1}{4}-} N} \prod_{i=1}^{6}\left\|u_{i}\right\|_{X^{0, \frac{1}{2}+}} \\
& \lesssim \frac{1 \wedge N_{\min }^{0+}}{N_{\max }^{0+} N^{2-}} \prod_{i=1}^{6}\left\|u_{i}\right\|_{X^{0, \frac{1}{2}+}} \cdot
\end{aligned}
$$

Case 2: $N_{4} \geq N_{5} \geq N \geq N_{6}$.

a. $N_{1} \geq N_{2} \geq N_{3} \geq N$.

This is handled like case 1a. without the factor $\left(\frac{N_{6}}{N}\right)^{\frac{1}{4}}$ and gives

$$
\begin{aligned}
C & \lesssim \frac{N_{3}^{\frac{3}{4}+} N_{6}^{\frac{1}{2}+}}{N_{1}^{\frac{3}{4}} N_{2}^{\frac{1}{4}} N_{4}^{\frac{1}{4}} N_{5}^{\frac{3}{4}} N^{\frac{5}{4}}} \prod_{i=1}^{6}\left\|u_{i}\right\|_{X^{0, \frac{1}{2}+}} \lesssim \frac{1 \wedge N_{\min }^{0+}}{N_{1}^{0+} N_{2}^{\frac{1}{4}-} N_{4}^{0+} N_{5}^{\frac{1}{2}-} N^{\frac{5}{4}}} \prod_{i=1}^{6}\left\|u_{i}\right\|_{X^{0, \frac{1}{2}+}} \\
& \lesssim \frac{1 \wedge N_{\min }^{0+}}{N_{\max }^{0+} N^{2-}} \prod_{i=1}^{6}\left\|u_{i}\right\|_{X^{0, \frac{1}{2}+}} \cdot
\end{aligned}
$$

b. $N_{1} \geq N_{2} \geq N \geq N_{3}$.

It can be handled like case $1 \mathrm{~b}$. without the factor $\left(\frac{N_{6}}{N}\right)^{\frac{1}{4}}$ leading to

$$
\begin{aligned}
C & \lesssim \frac{N_{3}^{\frac{1}{2}+} N_{6}^{\frac{1}{2}+}}{N_{1}^{\frac{3}{4}} N_{2}^{\frac{1}{4}} N_{4}^{\frac{1}{4}} N_{5}^{\frac{3}{4}} N} \prod_{i=1}^{6}\left\|u_{i}\right\|_{X^{0, \frac{1}{2}+}} \lesssim \frac{1 \wedge N_{\min }^{0+}}{N_{1}^{0+} N_{2}^{\frac{1}{2}-} N_{4}^{0+} N_{5}^{\frac{1}{2}-} N} \prod_{i=1}^{6}\left\|u_{i}\right\|_{X^{0, \frac{1}{2}+}} \\
& \lesssim \frac{1 \wedge N_{\min }^{0+}}{N_{\max }^{0+} N^{2-}} \prod_{i=1}^{6}\left\|u_{i}\right\|_{X^{0, \frac{1}{2}+}} \cdot
\end{aligned}
$$

c. $N_{1} \geq N \geq N_{2} \geq N_{3}$.

As in case 1c. without the factor $\left(\frac{N_{6}}{N}\right)^{\frac{1}{4}}$ we get

$$
\begin{aligned}
C & \lesssim \frac{N_{3}^{\frac{1}{2}+} N_{6}^{\frac{1}{2}+}}{N_{1}^{\frac{3}{4}} N_{2}^{\frac{1}{2}} N_{4}^{\frac{1}{4}} N_{5}^{\frac{3}{4}} N^{\frac{3}{4}}} \prod_{i=1}^{6}\left\|u_{i}\right\|_{X^{0, \frac{1}{2}+}} \lesssim \frac{1 \wedge N_{\min }^{0+}}{N_{1}^{\frac{3}{4}-} N_{4}^{\frac{1}{4}} N_{5}^{\frac{1}{4}-} N^{\frac{3}{4}}} \prod_{i=1}^{6}\left\|u_{i}\right\|_{X^{0, \frac{1}{2}+}} \\
& \lesssim \frac{1 \wedge N_{\min }^{0+}}{N_{\max }^{0+} N^{2-}} \prod_{i=1}^{6}\left\|u_{i}\right\|_{X^{0, \frac{1}{2}+}} \cdot
\end{aligned}
$$

Case 3: $N_{4} \gtrsim N \geq N_{5} \geq N_{6}$.

a. $N_{1} \geq N_{2} \geq N_{3} \geq N$. 
We have

$$
M\left(\xi_{1}, \ldots, \xi_{6}\right) \lesssim \prod_{i=1}^{3}\left(\frac{N_{i}}{N}\right)^{\frac{1}{4}} \prod_{i=1}^{6} N_{i}^{-1}
$$

Thus by Strichartz and Sobolev and the bilinear Strichartz estimate (11) we get

$$
\begin{aligned}
C & \lesssim \prod_{i=1}^{3}\left(\frac{N_{i}}{N}\right)^{\frac{1}{4}} \prod_{i=1}^{6} N_{i}^{-1}\left\|u_{1}\right\|_{L_{t}^{2} L_{x}^{6}}\left\|u_{2}\right\|_{L_{t}^{\infty} L_{x}^{3}}\left\|u_{3} u_{4}\right\|_{L_{t x}^{2}}\left\|u_{5}\right\|_{L_{x t}^{\infty}}\left\|u_{6}\right\|_{L_{x t}^{\infty}} \\
& \lesssim \prod_{i=1}^{3}\left(\frac{N_{i}}{N}\right)^{\frac{1}{4}} \prod_{i=1}^{6} N_{i}^{-1} \frac{N_{2}^{\frac{1}{2}} N_{3}^{1+}}{N_{4}^{\frac{1}{2}-}}\left(N_{5}^{\frac{3}{2}+}+N_{5}^{\frac{3}{2}-}\right)\left(N_{6}^{\frac{3}{2}+}+N_{6}^{\frac{3}{2}-}\right) \prod_{i=1}^{6}\left\|u_{i}\right\|_{X^{0, \frac{1}{2}+}} \\
& \lesssim \frac{N_{3}^{\frac{1}{4}+}\left(N_{5}^{\frac{1}{2}+}+N_{5}^{\frac{1}{2}-}\right)\left(N_{6}^{\frac{1}{2}+}+N_{6}^{\frac{1}{2}-}\right)}{N_{1}^{\frac{3}{4}} N_{2}^{\frac{1}{4}} N_{4}^{\frac{3}{2}-} N^{\frac{3}{4}}}\left\|u_{i}\right\|_{X^{0, \frac{1}{2}+}} \\
& \lesssim \frac{N^{1+}\left(1 \wedge N_{\min }^{0+}\right)}{N_{\max }^{0+} N^{3-}} \prod_{i=1}^{6}\left\|u_{i}\right\|_{X^{0, \frac{1}{2}+}} \\
& \lesssim \frac{1 \wedge N_{\min }^{0+} \prod_{i=1}^{6}\left\|u_{i}\right\|_{X^{0, \frac{1}{2}+}}}{N_{\max }^{0+} N^{2-}}
\end{aligned}
$$

b. $N_{1} \geq N_{2} \geq N \geq N_{3}$.

Similarly as in case a. without the factor $\left(\frac{N_{3}}{N}\right)^{\frac{1}{4}}$ we get

$$
\begin{aligned}
C & \lesssim \frac{N_{3}^{0+}\left(N_{5}^{\frac{1}{2}+}+N_{5}^{\frac{1}{2}-}\right)\left(N_{6}^{\frac{1}{2}+}+N_{6}^{\frac{1}{2}-}\right)}{N_{1}^{\frac{3}{4}} N_{2}^{\frac{1}{4}} N_{4}^{\frac{3}{2}-} N^{\frac{1}{2}}} \prod_{i=1}^{6}\left\|u_{i}\right\|_{X^{0, \frac{1}{2}+}} \\
& \lesssim \frac{N^{1+}\left(1 \wedge N_{\min }^{0+}\right)}{N_{\max }^{0+} N^{3-}} \prod_{i=1}^{6}\left\|u_{i}\right\|_{X^{0, \frac{1}{2}+}} \\
& \lesssim \frac{1 \wedge N_{\min }^{0+}}{N_{\max }^{0+} N^{2-}} \prod_{i=1}^{6}\left\|u_{i}\right\|_{X^{0, \frac{1}{2}+}}
\end{aligned}
$$

c. $N_{1} \geq N \geq N_{2} \geq N_{3}$.

The multiplier is estimated as follows using the mean value theorem

$$
\begin{aligned}
M\left(\xi_{1}, \ldots, \xi_{6}\right) & \lesssim\left|\frac{m\left(\xi_{1}+\xi_{2}+\xi_{3}\right)-m\left(\xi_{1}\right)}{m\left(\xi_{1}\right)}\right| \prod_{i=1}^{6}\left|\xi_{i}\right|^{-1} \\
& \sim \frac{\left|(\nabla m)\left(\xi_{1}\right)\left(\xi_{2}+\xi_{3}\right)\right|}{\left|m\left(\xi_{1}\right)\right|} \prod_{i=1}^{6}\left|\xi_{i}\right|^{-1} \lesssim \frac{N_{2}}{N_{1}} \prod_{i=1}^{6}\left|\xi_{i}\right|^{-1}
\end{aligned}
$$


Thus we get by Sobolev and Strichartz:

$$
\begin{aligned}
C & \lesssim \frac{N_{2}}{N_{1}} \prod_{i=1}^{6} N_{i}^{-1}\left\|u_{1}\right\|_{L_{t}^{2} L_{x}^{6}}\left\|u_{2}\right\|_{L_{t}^{\infty} L_{x}^{6}}\left\|u_{3}\right\|_{L_{t}^{\infty} L_{x}^{6+}}\left\|u_{4}\right\|_{L_{t}^{2} L_{x}^{6-}}\left\|u_{5}\right\|_{L_{t}^{\infty} L_{x}^{6}}\left\|u_{6}\right\|_{L_{t}^{\infty} L_{x}^{6+}} \\
& \lesssim \frac{N_{2}}{N_{1}} \prod_{i=1}^{6} N_{i}^{-1} N_{2} N_{3}^{1+} N_{5} N_{6}^{1+} \prod_{i=1}^{6}\left\|u_{i}\right\|_{X^{0, \frac{1}{2}+}} \\
& \lesssim \frac{N_{2} N_{3}^{0+} N_{6}^{0+}}{N_{1}^{2} N_{4}} \prod_{i=1}^{6}\left\|u_{i}\right\|_{X^{0, \frac{1}{2}+}} \\
& \lesssim \frac{1 \wedge N_{\min }^{0+}}{N_{\max }^{0+} N^{2-}} \prod_{i=1}^{6}\left\|u_{i}\right\|_{X^{0, \frac{1}{2}+}}
\end{aligned}
$$

Case 4: $N \gg N_{4} \geq N_{5} \geq N_{6}$.

a. $N_{1} \geq N_{2} \geq N_{3} \geq N$.

We have

$$
\begin{aligned}
C & \lesssim\left(\frac{N_{1}}{N}\right)^{\frac{1}{4}}\left(\frac{N_{2}}{N}\right)^{\frac{1}{4}}\left(\frac{N_{3}}{N}\right)^{\frac{1}{4}} \prod_{i=1}^{6} N_{i}^{-1}\left\|u_{1}\right\|_{L_{t}^{2} L_{x}^{6-}}\left\|u_{2}\right\|_{L_{t}^{2} L_{x}^{6-}} \prod_{i=3}^{6}\left\|u_{i}\right\|_{L_{t}^{\infty} L_{x}^{6+}} \\
& \lesssim\left(\frac{N_{1}}{N}\right)^{\frac{1}{4}}\left(\frac{N_{2}}{N}\right)^{\frac{1}{4}}\left(\frac{N_{3}}{N}\right)^{\frac{1}{4}} \prod_{i=1}^{6} N_{i}^{-1} N_{3}^{1+} N_{4}^{1+} N_{5}^{1+} N_{6}^{1+} \prod_{i=1}^{6}\left\|u_{i}\right\|_{X^{0, \frac{1}{2}+}} \\
& \lesssim \frac{N_{3}^{\frac{1}{4}+} N_{4}^{0+} N_{5}^{0+} N_{6}^{0+}}{N_{1}^{\frac{3}{4}} N_{2}^{\frac{3}{4}} N^{\frac{3}{4}}} \prod_{i=1}\left\|u_{i}\right\|_{X^{0, \frac{1}{2}+}} \\
& \lesssim \frac{1 \wedge N_{\min }^{0+}}{N_{\max }^{0+} N^{2-}} \prod_{i=1}^{6}\left\|u_{i}\right\|_{X^{0, \frac{1}{2}+}} \cdot
\end{aligned}
$$

b. $N_{1} \geq N_{2} \gtrsim N \gtrsim N_{3}$.

We get the same bound as in case a. without the factor $\left(\frac{N_{3}}{N}\right)^{\frac{1}{4}}$ leading to the same estimate.

c. $N_{1} \geq N \gg N_{2} \geq N_{3}$.

This case cannot occur, because $\sum_{i=1}^{6} \xi_{i}=0$.

4. Next we aim to show

$$
\int_{0}^{\delta}\left|\left\langle I\left(u^{3}\right)-(I u)^{3}, I\left(u^{2}\right)\right\rangle\right| d t \lesssim N^{-\frac{5}{2}+}\|\nabla I u\|_{X^{0, \frac{1}{2}+}}^{5},
$$

which follows from

$$
D=\left|\int_{0}^{\delta} \int_{*} M\left(\xi_{1}, \ldots, \xi_{5}\right) \prod_{i=1}^{5} \widehat{u}_{i}\left(\xi_{i}, t\right) d \xi_{1} \ldots d \xi_{5} d t\right| \lesssim N^{-\frac{5}{2}+} \prod_{i=1}^{5}\left\|u_{i}\right\|_{X^{0, \frac{1}{2}+}},
$$

where

$$
M\left(\xi_{1}, \ldots, \xi_{5}\right):=\frac{\left|m\left(\xi_{1}+\xi_{2}+\xi_{3}\right)-m\left(\xi_{1}\right) m\left(\xi_{2}\right) m\left(\xi_{3}\right)\right|}{m\left(\xi_{1}\right) m\left(\xi_{2}\right) m\left(\xi_{3}\right)} \frac{m\left(\xi_{4}+\xi_{5}\right)}{m\left(\xi_{4}\right) m\left(\xi_{5}\right)} \prod_{i=1}^{5}\left|\xi_{i}\right|^{-1} .
$$


Assume w.l.o.g. $N_{1} \geq N_{2} \geq N_{3}, N_{1} \geq N$ and $N_{4} \geq N_{5}$.

Case 1: $N_{4} \geq N_{5} \geq N$.

a. $N_{1} \geq N_{2} \geq N_{3} \geq N$.

Thus by Strichartz and Sobolev:

$$
\begin{aligned}
& D \lesssim \prod_{i=1}^{5}\left(\frac{N_{i}}{N}\right)^{\frac{1}{4}} \prod_{i=1}^{5} N_{i}^{-1}\left\|u_{1}\right\|_{L_{t}^{\infty} L_{x}^{3}}\left\|u_{2}\right\|_{L_{t}^{2} L_{x}^{6}}\left\|u_{3}\right\|_{L^{\infty} L_{x}^{6+}}\left\|u_{4}\right\|_{L_{t}^{2} L_{x}^{6-}}\left\|u_{5}\right\|_{L_{t}^{\infty} L_{x}^{6+}} \\
& \lesssim \prod_{i=1}^{5}\left(\frac{N_{i}}{N}\right)^{\frac{1}{4}} \prod_{i=1}^{5} N_{i}^{-1} N_{1}^{\frac{1}{2}} N_{3}^{1+} N_{5}^{1+} \prod_{i=1}^{5}\left\|u_{i}\right\|_{X^{0, \frac{1}{2}}+} \\
& \lesssim \frac{N_{3}^{\frac{1}{4}+} N_{5}^{\frac{1}{4}+}}{N_{1}^{\frac{1}{4}} N_{2}^{\frac{3}{4}} N_{4}^{\frac{3}{4}} N^{\frac{5}{4}}} \prod_{i=1}^{5}\left\|u_{i}\right\|_{X^{0, \frac{1}{2}}+} \\
& \lesssim \frac{1 \wedge N_{\min }^{0+}}{N_{\max }^{0+} N^{\frac{5}{2}-}} \prod_{i=1}^{5}\left\|u_{i}\right\|_{X^{0, \frac{1}{2}+}}
\end{aligned}
$$

b. $N_{1} \geq N_{2} \geq N \geq N_{3}$.

As in case a. (without the factor $\left(\frac{N_{3}}{N}\right)^{\frac{1}{4}}$ ) we get the same estimate.

c. $N_{1} \geq N \geq N_{2} \geq N_{3}$ and $N_{1} \gg N_{2}$.

The multiplier is estimated as follows:

$$
\begin{aligned}
\left|\frac{m\left(\xi_{1}+\xi_{2}+\xi_{3}\right)-m\left(\xi_{1}\right) m\left(\xi_{2}\right) m\left(\xi_{3}\right)}{m\left(\xi_{1}\right) m\left(\xi_{2}\right) m\left(\xi_{3}\right)}\right| & =\left|\frac{m\left(\xi_{1}+\xi_{2}+\xi_{3}\right)-m\left(\xi_{1}\right)}{m\left(\xi_{1}\right)}\right| \\
& \sim\left|\frac{(\nabla m)\left(\xi_{1}\right)\left(\xi_{2}+\xi_{3}\right)}{m\left(\xi_{1}\right)}\right| \lesssim \frac{N_{2}}{N_{1}}
\end{aligned}
$$

Thus as in case a. we get

$$
\begin{aligned}
D & \lesssim \frac{N_{2}}{N_{1}}\left(\frac{N_{4}}{N}\right)^{\frac{1}{4}}\left(\frac{N_{5}}{N}\right)^{\frac{1}{4}} \prod_{i=1}^{5} N_{i}^{-1} N_{1}^{\frac{1}{2}} N_{3}^{1+} N_{5}^{1+} \prod_{i=1}^{5}\left\|u_{i}\right\|_{X^{0, \frac{1}{2}+}} \\
& \lesssim \frac{N_{3}^{0+} N_{5}^{\frac{1}{4}+}}{N_{1}^{\frac{3}{2}} N_{4}^{\frac{3}{4}} N^{\frac{1}{2}}} \prod_{i=1}^{5}\left\|u_{i}\right\|_{X^{0, \frac{1}{2}+}} \\
& \lesssim \frac{1 \wedge N_{\min }^{0+}}{N_{\max }^{0+} N^{\frac{5}{2}}-} \prod_{i=1}^{5}\left\|u_{i}\right\|_{X^{0, \frac{1}{2}+}} \cdot
\end{aligned}
$$

Case 2: $N_{4} \gtrsim N \geq N_{5}$.

a. $N_{1} \geq N_{2} \geq N_{3} \geq N$

As in case 1a. (without the factor $\left(\frac{N_{5}}{N}\right)^{\frac{1}{4}}$ ) we get the same estimate.

b. $N_{1} \geq N_{2} \geq N \geq N_{3}$.

As in case 1a. (without the factor $\left(\frac{N_{3}}{N}\right)^{\frac{1}{4}}\left(\frac{N_{5}}{N}\right)^{\frac{1}{4}}$ ) we get the same estimate.

c. $N_{1} \geq N \geq N_{2} \geq N_{3}$.

As in case 1c. (without the factor $\left(\frac{N_{5}}{N}\right)^{\frac{1}{4}}$ ) we get the same estimate.

Case 3: $N \gg N_{4} \geq N_{5} \Rightarrow N_{1} \sim N_{2} \gtrsim N$, because $\sum_{i=1}^{5} \xi_{i}=0$.

a. $N_{1} \sim N_{2} \geq N_{3} \geq N$. 
By Strichartz and Sobolev and the bilinear Strichartz estimate (11) we get

$$
\begin{aligned}
D & \lesssim \prod_{i=1}^{3}\left(\frac{N_{i}}{N}\right)^{\frac{1}{4}} \prod_{i=1}^{5} N_{i}^{-1}\left\|u_{1} u_{3}\right\|_{L_{x t}^{2}}\left\|u_{2}\right\|_{L_{t}^{2} L_{x}^{6-}}\left\|u_{4}\right\|_{L_{t}^{\infty} L_{x}^{6}}\left\|u_{5}\right\|_{L_{t}^{\infty} L_{x}^{6+}} \\
& \lesssim \prod_{i=1}^{3}\left(\frac{N_{i}}{N}\right)^{\frac{1}{4}} \prod_{i=1}^{5} N_{i}^{-1} \frac{N_{3}^{1+}}{N_{1}^{\frac{1}{2}-}} N_{4} N_{5}^{1+} \prod_{i=1}^{5}\left\|u_{i}\right\|_{X^{0, \frac{1}{2}+}} \\
& \lesssim \frac{N_{3}^{\frac{1}{4}+} N_{5}^{0+}}{N_{1}^{\frac{5}{4}-} N_{2}^{\frac{3}{4}} N^{\frac{3}{4}}} \prod_{i=1}^{5}\left\|u_{i}\right\|_{X^{0, \frac{1}{2}}} \lesssim \frac{1 \wedge N_{\min }^{0+}}{N_{\max }^{0+} N^{\frac{5}{2}}-} \prod_{i=1}^{5}\left\|u_{i}\right\|_{X^{0, \frac{1}{2}+}}
\end{aligned}
$$

b. $N_{1} \sim N_{2} \gtrsim N \geq N_{3}$.

Without the factor $\left.\left(\frac{N_{3}}{N}\right)^{\frac{1}{4}}\right)$ we get the same estimate as in case a.

5. Next we want to show

$$
\int_{0}^{\delta}\left|\left\langle I\left(u^{2}\right)-(I u)^{2}, I\left(u^{3}\right)\right\rangle\right| d t \lesssim N^{-\frac{5}{2}+}\|\nabla I u\|_{X^{0, \frac{1}{2}+}}^{5} .
$$

We have to prove

$$
E=\left|\int_{0}^{\delta} \int_{*} M\left(\xi_{1}, \ldots, \xi_{5}\right) \prod_{i=1}^{5} \widehat{u}_{i}\left(\xi_{i}, t\right) d \xi_{1} \ldots d \xi_{5} d t\right| \lesssim N^{-\frac{5}{2}+} \prod_{i=1}^{5}\left\|u_{i}\right\|_{X^{0, \frac{1}{2}+}},
$$

where

$$
M\left(\xi_{1}, \ldots, \xi_{5}\right):=\frac{\left|m\left(\xi_{1}+\xi_{2}\right)-m\left(\xi_{1}\right) m\left(\xi_{2}\right)\right|}{m\left(\xi_{1}\right) m\left(\xi_{2}\right)} \frac{m\left(\xi_{3}+\xi_{4}+\xi_{5}\right)}{m\left(\xi_{3}\right) m\left(\xi_{4}\right) m\left(\xi_{5}\right)} \prod_{i=1}^{5}\left|\xi_{i}\right|^{-1} .
$$

Assume w.l.o.g. $N_{3} \geq N_{4} \geq N_{5}, N_{1} \geq N_{2}$ and $N_{1} \geq N$.

Case 1: $N_{3} \geq N_{4} \geq N_{5} \geq N$.

a. $N_{1} \geq N_{2} \gtrsim N$.

This case can be handled exactly as in 4 . case $1 \mathrm{a}$.

b. $N_{1} \geq N \gg N_{2}$.

We get by use of the mean value theorem for the first fraction and estimating similarly as in 4 . case 1 a. (interchanging the roles of $u_{2}$ and $u_{3}$ ):

$$
\begin{aligned}
E & \lesssim \frac{N_{2}}{N_{1}} \prod_{i=3}^{5}\left(\frac{N_{i}}{N}\right)^{\frac{1}{4}} \prod_{i=1}^{5} N_{i}^{-1} N_{1}^{\frac{1}{2}} N_{2}^{1+} N_{5}^{1+} \prod_{i=1}^{5}\left\|u_{i}\right\|_{X^{0, \frac{1}{2}+}} \\
& \lesssim \frac{N_{2}^{1+} N_{5}^{\frac{1}{4}+}}{N_{1}^{\frac{3}{2}} N_{3}^{\frac{3}{4}} N_{4}^{\frac{3}{4}} N^{\frac{3}{4}}} \prod_{i=1}^{5}\left\|u_{i}\right\|_{X^{0, \frac{1}{2}+}} \\
& \lesssim \frac{1 \wedge N_{\min }^{0+} \prod_{i=1}^{5}\left\|u_{i}\right\|_{X^{0, \frac{1}{2}+}} .}{N_{\max }^{0+} N^{\frac{5}{2}-}} \prod_{i=1}
\end{aligned}
$$

Case 2: $N_{3} \geq N_{4} \geq N \geq N_{5}$. a. $N_{1} \geq N_{2} \gtrsim N$. 
By Strichartz and Sobolev we get

$$
\begin{aligned}
E & \lesssim \prod_{i=1}^{4}\left(\frac{N_{i}}{N}\right)^{\frac{1}{4}} \prod_{i=1}^{5} N_{i}^{-1}\left\|u_{1}\right\|_{L_{t}^{\infty} L_{x}^{3}}\left\|u_{2}\right\|_{L_{t}^{2} L_{x}^{6-}}\left\|u_{3}\right\|_{L_{t}^{2} L_{x}^{6}}\left\|u_{4}\right\|_{L_{t}^{\infty} L_{x}^{6}}\left\|u_{5}\right\|_{L_{t}^{\infty} L_{x}^{6+}} \\
& \lesssim \prod_{i=1}^{4}\left(\frac{N_{i}}{N}\right)^{\frac{1}{4}} \prod_{i=1}^{5} N_{i}^{-1} N_{1}^{\frac{1}{2}} N_{4} N_{5}^{1+} \prod_{i=1}^{5}\left\|u_{i}\right\|_{X^{0, \frac{1}{2}+}} \\
& \lesssim \frac{N_{4}^{\frac{1}{4}} N_{5}^{0+}}{N_{1}^{\frac{1}{4}} N_{2}^{\frac{3}{4}} N_{3}^{\frac{3}{4}} N} \prod_{i=1}^{5}\left\|u_{i}\right\|_{X^{0, \frac{1}{2}+}} \\
& \lesssim \frac{1 \wedge N_{\min }^{0+}}{N_{\max }^{0+} N^{\frac{5}{2}}-} \prod_{i=1}^{5}\left\|u_{i}\right\|_{X^{0, \frac{1}{2}+}}
\end{aligned}
$$

b. $N_{1} \geq N_{2} \gg N_{2}$.

By the mean value theorem we get as in a. (slightly modified):

$$
\begin{aligned}
E & \lesssim \frac{N_{2}}{N_{1}}\left(\frac{N_{3}}{N}\right)^{\frac{1}{4}}\left(\frac{N_{4}}{N}\right)^{\frac{1}{4}} \prod_{i=1}^{5} N_{i}^{-1} N_{1}^{\frac{1}{2}} N_{2}^{0+} N_{4}^{1-} N_{5}^{1+} \prod_{i=1}^{5}\left\|u_{i}\right\|_{X^{0, \frac{1}{2}}} \\
& \lesssim \frac{N_{2}^{0+} N_{4}^{\frac{1}{4}-} N_{5}^{0+}}{N_{1}^{\frac{3}{2}} N_{3}^{\frac{3}{4}} N^{\frac{1}{2}}} \prod_{i=1}^{5}\left\|u_{i}\right\|_{X^{0, \frac{1}{2}+}} \\
& \lesssim \frac{1 \wedge N_{\min }^{0+}}{N_{\max }^{0+} N^{\frac{5}{2}}-} \prod_{i=1}^{5}\left\|u_{i}\right\|_{X^{0, \frac{1}{2}+}} .
\end{aligned}
$$

Case 3: $N_{3} \gtrsim N \geq N_{4} \geq N_{5}$.

a. $N_{1} \geq N_{2} \geq N$.

The second fraction is bounded, so that as in case $2 \mathrm{a}$. we get

$$
\begin{aligned}
E & \lesssim\left(\frac{N_{1}}{N}\right)^{\frac{1}{4}}\left(\frac{N_{2}}{N}\right)^{\frac{1}{4}} \prod_{i=1}^{5} N_{i}^{-1} N_{1}^{\frac{1}{2}} N_{4} N_{5}^{1+} \prod_{i=1}^{5}\left\|u_{i}\right\|_{X^{0, \frac{1}{2}+}} \\
& \lesssim \frac{N_{5}^{0+}}{N_{1}^{\frac{1}{4}} N_{2}^{\frac{3}{4}} N_{3} N^{\frac{1}{2}}} \prod_{i=1}^{5}\left\|u_{i}\right\|_{X^{0, \frac{1}{2}+}} \\
& \lesssim \frac{1 \wedge N_{\min }^{0+}}{N_{\max }^{0+} N^{\frac{5}{2}}-} \prod_{i=1}^{5}\left\|u_{i}\right\|_{X^{0, \frac{1}{2}+}} \cdot
\end{aligned}
$$

b. $N_{1} \geq N \geq N_{2}$.

Using the mean value theorem and interchanging the roles of $u_{1}$ and $u_{2}$ in case 
a. gives

$$
\begin{aligned}
E & \lesssim \frac{N_{2}}{N_{1}} \prod_{i=1}^{5} N_{i}^{-1} N_{2}^{\frac{1}{2}} N_{4} N_{5}^{1+} \prod_{i=1}^{5}\left\|u_{i}\right\|_{X^{0, \frac{1}{2}+}} \\
& \lesssim \frac{N_{2}^{\frac{1}{2}} N_{5}^{0+}}{N_{1}^{2} N_{3}} \prod_{i=1}^{5}\left\|u_{i}\right\|_{X^{0, \frac{1}{2}+}} \\
& \lesssim \frac{1 \wedge N_{\min }^{0+}}{N_{\max }^{0+} N^{\frac{5}{2}}-} \prod_{i=1}^{5}\left\|u_{i}\right\|_{X^{0, \frac{1}{2}+}}
\end{aligned}
$$

Case 4: $N \gg N_{3} \geq N_{4} \geq N_{5} \Rightarrow N_{1} \sim N_{2} \gtrsim N$, because $\sum_{i=1}^{5} \xi_{i}=0$.

This gives by Strichartz, Sobolev and the bilinear Strichartz estimate (10)

$$
\begin{aligned}
E & \lesssim\left(\frac{N_{1}}{N}\right)^{\frac{1}{4}}\left(\frac{N_{2}}{N}\right)^{\frac{1}{4}} \prod_{i=1}^{5} N_{i}^{-1}\left\|u_{1} u_{3}\right\|_{L_{x t}^{2}}\left\|u_{2}\right\|_{L_{t}^{2} L_{x}^{6-}}\left\|u_{4}\right\|_{L_{t}^{\infty} L_{x}^{6}}\left\|u_{5}\right\|_{L_{t}^{\infty} L_{x}^{6+}} \\
& \lesssim\left(\frac{N_{1}}{N}\right)^{\frac{1}{4}}\left(\frac{N_{2}}{N}\right)^{\frac{1}{4}} \prod_{i=1}^{5} N_{i}^{-1} \frac{N_{3}}{N_{1}^{\frac{1}{2}}} N_{4} N_{5}^{1+} \prod_{i=1}^{5}\left\|u_{i}\right\|_{X^{0, \frac{1}{2}+}} \\
& \lesssim \frac{N_{5}^{0+}}{N_{1}^{\frac{5}{4}} N_{2}^{\frac{3}{4}} N^{\frac{1}{2}}} \prod_{i=1}^{5}\left\|u_{i}\right\|_{X^{0, \frac{1}{2}+}} \\
& \lesssim \frac{1 \wedge N_{\min }^{0+}}{N_{\max }^{0+} N^{\frac{5}{2}-}} \prod_{i=1}^{5}\left\|u_{i}\right\|_{X^{0, \frac{1}{2}+}} \cdot
\end{aligned}
$$

6. Next we want to prove

$$
\int_{0}^{\delta}\left|\left\langle I\left(u^{3}\right)-(I u)^{3}, I u\right\rangle\right| d t \lesssim N^{-3+}\|\nabla I u\|_{X^{0, \frac{1}{2}+}}^{4}
$$

which follows from

$$
F=\left|\int_{0}^{\delta} \int_{*} M\left(\xi_{1}, \ldots, \xi_{4}\right) \prod_{i=1}^{4} \widehat{u}_{i}\left(\xi_{i}, t\right) d \xi_{1} \ldots d \xi_{4} d t\right| \lesssim N^{-3+} \prod_{i=1}^{4}\left\|u_{i}\right\|_{X^{0, \frac{1}{2}+}}
$$

where

$$
M\left(\xi_{1}, \xi_{2}, \xi_{3}\right):=\frac{\left|m\left(\xi_{1}+\xi_{2}+\xi_{3}\right)-m\left(\xi_{1}\right) m\left(\xi_{2}\right) m\left(\xi_{3}\right)\right|}{m\left(\xi_{1}\right) m\left(\xi_{2}\right) m\left(\xi_{3}\right)} \prod_{i=1}^{4}\left|\xi_{i}\right|^{-1}
$$

Assume w.l.o.g. $N_{1} \geq N_{2} \geq N_{3}$ and $N_{1} \geq N$.

Case 1: $N \gg N_{4}$.

a. $N_{1} \geq N_{2} \geq N_{3} \geq N$. 
By the bilinear Strichartz refinement (11) we get

$$
\begin{aligned}
E & \lesssim \prod_{i=1}^{3}\left(\frac{N_{i}}{N}\right)^{\frac{1}{4}} \prod_{i=1}^{4} N_{i}^{-1}\left\|u_{2} u_{4}\right\|_{L_{x t}^{2}}\left\|u_{1} u_{3}\right\|_{L_{x t}^{2}} \\
& \lesssim \prod_{i=1}^{3}\left(\frac{N_{i}}{N}\right)^{\frac{1}{4}} \prod_{i=1}^{4} N_{i}^{-1} \frac{N_{4}^{1+}}{N_{2}^{\frac{1}{2}-}} \frac{N_{3}^{1+}}{N_{1}^{\frac{1}{2}}-} \prod_{i=1}^{4}\left\|u_{i}\right\|_{X^{0, \frac{1}{2}+}} \\
& \lesssim \frac{N_{3}^{\frac{1}{4}+} N_{4}^{0+}}{N_{1}^{\frac{5}{4}-} N_{2}^{\frac{5}{4}-} N^{\frac{3}{4}}} \prod_{i=1}^{4}\left\|u_{i}\right\|_{X^{0, \frac{1}{2}+}} \lesssim \frac{1 \wedge N_{\min }^{0+}}{N_{\max }^{0+} N^{3-}} \prod_{i=1}^{4}\left\|u_{i}\right\|_{X^{0, \frac{1}{2}+}}
\end{aligned}
$$

b. $N_{1} \geq N_{2} \gtrsim N \geq N_{3}$.

Similarly as in case a. we get

$$
\begin{aligned}
E & \lesssim \prod_{i=1}^{2}\left(\frac{N_{i}}{N}\right)^{\frac{1}{4}} \prod_{i=1}^{4} N_{i}^{-1} \frac{N_{4}^{1+}}{N_{2}^{\frac{1}{2}-}} \frac{N_{3}^{1+}}{N_{1}^{\frac{1}{2}}-} \prod_{i=1}^{4}\left\|u_{i}\right\|_{X^{0, \frac{1}{2}+}} \\
& \lesssim \frac{N_{3}^{0+} N_{4}^{0+}}{N_{1}^{\frac{5}{4}-} N_{2}^{\frac{5}{4}-} N^{\frac{1}{2}}} \prod_{i=1}^{4}\left\|u_{i}\right\|_{X^{0, \frac{1}{2}+}} \lesssim \frac{1 \wedge N_{\min }^{0+}}{N_{\max }^{0+} N^{3-}} \prod_{i=1}^{4}\left\|u_{i}\right\|_{X^{0, \frac{1}{2}+}}
\end{aligned}
$$

c. $N_{1} \geq N \gg N_{2} \geq N_{3}$.

This case does not occur, because $\sum_{i=1}^{4} \xi_{i}=0$.

Case 2: $N_{4} \gtrsim N$.

a. $N_{1} \geq N_{2} \geq N_{3} \geq N$ and

b. $N_{1} \geq N_{2} \gtrsim N \geq N_{3}$ can be treated as in case 1 .

c. $N_{1} \geq N \gg N_{2} \geq N_{3} \Rightarrow N_{1} \sim N_{4}$.

By the mean value theorem and the bilinear Strichartz refinement (11) we get

$$
\begin{aligned}
E & \lesssim \frac{N_{2}}{N_{1}} \prod_{i=1}^{4} N_{i}^{-1}\left\|u_{1} u_{2}\right\|_{L_{x t}^{2}}\left\|u_{3} u_{4}\right\|_{L_{x t}^{2}} \\
& \lesssim \frac{N_{2}}{N_{1}} \prod_{i=1}^{4} N_{i}^{-1} \frac{N_{2}}{N_{1}^{\frac{1}{2}}} \frac{N_{3}^{1+}}{N_{4}^{\frac{1}{2}-}} \prod_{i=1}^{4}\left\|u_{i}\right\|_{X^{0, \frac{1}{2}+}} \\
& \lesssim \frac{N_{2} N_{3}^{0+}}{N_{1}^{\frac{5}{2}} N_{4}^{\frac{3}{2}-}} \prod_{i=1}^{4}\left\|u_{i}\right\|_{X^{0, \frac{1}{2}+}} \lesssim \frac{1 \wedge N_{\min }^{0+} \prod_{N_{\max }^{0+} N^{3-}}^{4}\left\|u_{i}\right\|_{X^{0, \frac{1}{2}+}}}{}
\end{aligned}
$$

7. Next we prove

$$
\int_{0}^{\delta}\left|\left\langle I\left(u^{2}\right)-(I u)^{2},(I u)^{2}\right\rangle\right| d t \lesssim N^{-3+}\|\nabla I u\|_{X^{0, \frac{1}{2}+}}^{4}
$$

which follows from

$$
F=\left|\int_{0}^{\delta} \int_{*} M\left(\xi_{1}, \ldots, \xi_{4}\right) \prod_{i=1}^{4} \widehat{u_{i}}\left(\xi_{i}, t\right) d \xi_{1} \ldots d \xi_{4} d t\right| \lesssim N^{-3+} \prod_{i=1}^{4}\left\|u_{i}\right\|_{X^{0, \frac{1}{2}+}},
$$


where

$$
M\left(\xi_{1}, \xi_{2}, \xi_{3}\right):=\frac{\left|m\left(\xi_{1}+\xi_{2}\right)-m\left(\xi_{1}\right) m\left(\xi_{2}\right)\right|}{m\left(\xi_{1}\right) m\left(\xi_{2}\right)} \frac{m\left(\xi_{3}+\xi_{4}\right)}{m\left(\xi_{3}\right) m\left(\xi_{4}\right)} \prod_{i=1}^{4}\left|\xi_{i}\right|^{-1} .
$$

Assume w.l.o.g. $N_{1} \geq N_{2}, N_{1} \geq N$ and $N_{3} \geq N_{4}$.

Case 1: $N_{3} \geq N_{4} \gtrsim N$.

a. $N_{1} \geq N_{2} \geq N$.

By the bilinear Strichartz refinement (11) we get

$$
\begin{aligned}
F & \lesssim \prod_{i=1}^{4}\left(\frac{N_{i}}{N}\right)^{\frac{1}{4}} \prod_{i=1}^{4} N_{i}^{-1}\left\|u_{1} u_{2}\right\|_{L_{x t}^{2}}\left\|u_{3} u_{4}\right\|_{L_{x t}^{2}} \\
& \lesssim \prod_{i=1}^{4}\left(\frac{N_{i}}{N}\right)^{\frac{1}{4}} \prod_{i=1}^{4} N_{i}^{-1} \frac{N_{2}^{1+}}{N_{1}^{\frac{1}{2}-}} \frac{N_{4}^{1+}}{N_{3}^{\frac{1}{2}-}} \prod_{i=1}^{4}\left\|u_{i}\right\|_{X^{0, \frac{1}{2}+}} \\
& \lesssim \frac{N_{2}^{\frac{1}{4}+} N_{4}^{\frac{1}{4}+}}{N_{1}^{\frac{5}{4}-} N_{3}^{\frac{5}{4}-} N} \prod_{i=1}^{4}\left\|u_{i}\right\|_{X^{0, \frac{1}{2}}+} \lesssim \frac{1 \wedge N_{\min }^{0+}}{N_{\max }^{0+} N^{3-}} \prod_{i=1}^{4}\left\|u_{i}\right\|_{X^{0, \frac{1}{2}+}}
\end{aligned}
$$

b. $N_{1} \geq N \geq N_{2}$.

By the mean value theorem we get

$$
\begin{aligned}
F & \lesssim \frac{N_{2}}{N_{1}}\left(\frac{N_{3}}{N}\right)^{\frac{1}{4}}\left(\frac{N_{4}}{N}\right)^{\frac{1}{4}} \prod_{i=1}^{4} N_{i}^{-1} \frac{N_{2}^{1+}}{N_{1}^{\frac{1}{2}-}} \frac{N_{4}^{1+}}{N_{3}^{\frac{1}{2}}-} \prod_{i=1}^{4}\left\|u_{i}\right\|_{X^{0, \frac{1}{2}+}} \\
& \lesssim \frac{N_{2}^{1+} N_{4}^{\frac{1}{4}+}}{N_{1}^{\frac{5}{2}-} N_{3}^{\frac{5}{4}-} N^{\frac{1}{2}}} \prod_{i=1}^{4}\left\|u_{i}\right\|_{X^{0, \frac{1}{2}+}} \lesssim \frac{1 \wedge N_{\min }^{0+}}{N_{\max }^{0+} N^{3-}} \prod_{i=1}^{4}\left\|u_{i}\right\|_{X^{0, \frac{1}{2}+}}
\end{aligned}
$$

Case 2: $N_{3} \gtrsim N \geq N_{4}$ and $N_{3} \gg N_{4}$.

a. $N_{1} \geq N_{2} \geq N$.

We get similarly as in case $1 \mathrm{a}$.

$$
\begin{aligned}
F & \lesssim \prod_{i=1}^{2}\left(\frac{N_{i}}{N}\right)^{\frac{1}{4}} \prod_{i=1}^{4} N_{i}^{-1} \frac{N_{2}^{1+}}{N_{1}^{\frac{1}{2}-}} \frac{N_{4}^{1+}}{N_{3}^{\frac{1}{2}-}} \prod_{i=1}^{4}\left\|u_{i}\right\|_{X^{0, \frac{1}{2}+}} \\
& \lesssim \frac{N_{2}^{\frac{1}{4}+} N_{4}^{0+}}{N_{1}^{\frac{5}{4}-} N_{3}^{\frac{3}{2}-} N^{\frac{1}{2}}} \prod_{i=1}^{4}\left\|u_{i}\right\|_{X^{0, \frac{1}{2}+}} \\
& \lesssim \frac{1 \wedge N_{\min }^{0+}}{N_{\max }^{0+} N^{3-}} \prod_{i=1}^{4}\left\|u_{i}\right\|_{X^{0, \frac{1}{2}+}} .
\end{aligned}
$$

b. $N_{1} \geq N \geq N_{2}$.

By the mean value theorem we get similarly as in case 1a:

$$
\begin{aligned}
F & \lesssim \frac{N_{2}}{N_{1}} \prod_{i=1}^{4} N_{i}^{-1} \frac{N_{2}^{1+}}{N_{1}^{\frac{1}{2}-}} \frac{N_{4}^{1+}}{N_{3}^{\frac{1}{2}-}} \prod_{i=1}^{4}\left\|u_{i}\right\|_{X^{0, \frac{1}{2}+}} \\
& \lesssim \frac{N_{2}^{1+} N_{4}^{0+}}{N_{1}^{\frac{5}{2}-} N_{3}^{\frac{3}{2}-}} \prod_{i=1}^{4}\left\|u_{i}\right\|_{X^{0, \frac{1}{2}+}} \lesssim \frac{1 \wedge N_{\min }^{0+}}{N_{\max }^{0+} N^{3-}} \prod_{i=1}^{4}\left\|u_{i}\right\|_{X^{0, \frac{1}{2}+}} \cdot
\end{aligned}
$$


Case 3: $N \gg N_{3} \geq N_{4} \Rightarrow N_{1} \sim N_{2} \gtrsim N$, because $\sum_{i=1}^{4} \xi_{i}=0$.

We get by (11):

$$
\begin{aligned}
F & \lesssim\left(\frac{N_{1}}{N}\right)^{\frac{1}{4}}\left(\frac{N_{2}}{N}\right)^{\frac{1}{4}} \prod_{i=1}^{4} N_{i}^{-1}\left\|u_{1} u_{3}\right\|_{L_{x t}^{2}}\left\|u_{2} u_{4}\right\|_{L_{x t}^{2}} \\
& \lesssim\left(\frac{N_{1}}{N}\right)^{\frac{1}{4}}\left(\frac{N_{2}}{N}\right)^{\frac{1}{4}} \prod_{i=1}^{4} N_{i}^{-1} \frac{N_{3}}{N_{1}^{\frac{1}{2}}} \frac{N_{4}^{1+}}{N_{2}^{\frac{1}{2}}-} \prod_{i=1}^{4}\left\|u_{i}\right\|_{X^{0, \frac{1}{2}+}} \\
& \lesssim \frac{N_{4}^{0+}}{N_{1}^{\frac{5}{4}} N_{2}^{\frac{5}{4}-} N^{\frac{1}{2}}} \prod_{i=1}^{4}\left\|u_{i}\right\|_{X^{0, \frac{1}{2}+}} \lesssim \frac{1 \wedge N_{\min }^{0+}}{N_{\max }^{0+} N^{3-}} \prod_{i=1}^{4}\left\|u_{i}\right\|_{X^{0, \frac{1}{2}+}} \cdot
\end{aligned}
$$

8. Finally we prove

$$
\int_{0}^{\delta}\left|\left\langle I\left(u^{2}\right)-(I u)^{2}, I u\right\rangle\right| d t \lesssim N^{-\frac{5}{2}+} \delta^{\frac{1}{2}}\|\nabla I u\|_{X^{0, \frac{1}{2}+}}^{3},
$$

which follows from

$G=\left|\int_{0}^{\delta} \int_{*} M\left(\xi_{1}, \ldots, \xi_{4}\right) \prod_{i=1}^{3} \widehat{u}_{i}\left(\xi_{i}, t\right) d \xi_{1} d \xi_{2} d \xi_{3} d t\right| \lesssim N^{-\frac{5}{2}+} \delta^{\frac{1}{2}} \prod_{i=1}^{3}\left\|u_{i}\right\|_{X^{0, \frac{1}{2}+}}$,

where

$$
M\left(\xi_{1}, \xi_{2}, \xi_{3}\right):=\frac{\left|m\left(\xi_{1}+\xi_{2}\right)-m\left(\xi_{1}\right) m\left(\xi_{2}\right)\right|}{m\left(\xi_{1}\right) m\left(\xi_{2}\right)} \prod_{i=1}^{3}\left|\xi_{i}\right|^{-1} .
$$

Assume w.l.o.g. $N_{1} \geq N_{2}$ and $N_{1} \geq N$.

Case 1: $N_{3} \gtrsim N$.

a. $N_{1} \geq N_{2} \gtrsim N$.

By Strichartz and Sobolev we get:

$$
\begin{aligned}
G & \lesssim\left(\frac{N_{1}}{N}\right)^{\frac{1}{4}}\left(\frac{N_{2}}{N}\right)^{\frac{1}{4}} \prod_{i=1}^{3} N_{i}^{-1}\left\|u_{1}\right\|_{L_{t}^{2} L_{x}^{6}}\left\|u_{2}\right\|_{L_{t}^{\infty} L_{x}^{3}}\left\|u_{3}\right\|_{L_{x t}^{2}} \\
& \lesssim\left(\frac{N_{1}}{N}\right)^{\frac{1}{4}}\left(\frac{N_{2}}{N}\right)^{\frac{1}{4}} \prod_{i=1}^{3} N_{i}^{-1} N_{2}^{\frac{1}{2}} \delta^{\frac{1}{2}} \prod_{i=1}^{3}\left\|u_{i}\right\|_{X^{0, \frac{1}{2}+}} \\
& \lesssim \frac{1}{N_{1}^{\frac{3}{4}} N_{3} N_{2}^{\frac{1}{4}} N^{\frac{1}{2}}} \delta^{\frac{1}{2}} \prod_{i=1}^{3}\left\|u_{i}\right\|_{X^{0, \frac{1}{2}}+} \lesssim \frac{1 \wedge N_{\min }^{0+}}{N_{\max }^{0+} N^{\frac{5}{2}-}} \delta^{\frac{1}{2}} \prod_{i=1}^{3}\left\|u_{i}\right\|_{X^{0, \frac{1}{2}+}} \cdot
\end{aligned}
$$

b. $N_{1} \geq N \geq N_{2}$ and $N_{1} \gg N_{2}$.

Using the mean value theorem we get

$$
\begin{aligned}
G & \lesssim \frac{N_{2}}{N_{1}} \prod_{i=1}^{3} N_{i}^{-1} N_{2}^{\frac{1}{2}} \delta^{\frac{1}{2}} \prod_{i=1}^{3}\left\|u_{i}\right\|_{X^{0, \frac{1}{2}+}} \\
& \lesssim \frac{N_{2}^{\frac{1}{2}}}{N_{1}^{2} N_{3}} \delta^{\frac{1}{2}} \prod_{i=1}^{3}\left\|u_{i}\right\|_{X^{0, \frac{1}{2}+}} \lesssim \frac{1 \wedge N_{\min }^{0+}}{N_{\max }^{0+} N^{\frac{5}{2}-}} \delta^{\frac{1}{2}} \prod_{i=1}^{3}\left\|u_{i}\right\|_{X^{0, \frac{1}{2}+}}
\end{aligned}
$$


Case 2: $N \gg N_{3} \Rightarrow N_{1} \sim N_{2} \gtrsim N$, because $\sum_{i=1}^{3}=0$.

By (11) we get the estimate

$$
\begin{aligned}
G & \lesssim\left(\frac{N_{1}}{N}\right)^{\frac{1}{4}}\left(\frac{N_{2}}{N}\right)^{\frac{1}{4}} \prod_{i=1}^{3} N_{i}^{-1}\left\|u_{1} u_{3}\right\|_{L_{t x}^{3}}\left\|u_{2}\right\|_{L_{x t}^{2}} \\
& \lesssim\left(\frac{N_{1}}{N}\right)^{\frac{1}{4}}\left(\frac{N_{2}}{N}\right)^{\frac{1}{4}} \prod_{i=1}^{3} N_{i}^{-1} \frac{N_{3}^{1+}}{N_{1}^{\frac{1}{2}-}} \delta^{\frac{1}{2}} \prod_{i=1}^{3}\left\|u_{i}\right\|_{X^{0, \frac{1}{2}+}} \\
& \lesssim \frac{N_{3}^{0+}}{N_{1}^{\frac{5}{4}-} N_{2}^{\frac{3}{4}} N^{\frac{1}{2}}} \delta^{\frac{1}{2}} \prod_{i=1}^{3}\left\|u_{i}\right\|_{X^{0, \frac{1}{2}+}} \lesssim \frac{1 \wedge N_{\min }^{0+}}{N_{\max }^{0+} N^{\frac{5}{2}-}} \delta^{\frac{1}{2}} \prod_{i=1}^{3}\left\|u_{i}\right\|_{X^{0, \frac{1}{2}+}} \cdot
\end{aligned}
$$

This completes the estimates for the increment of $E(I u)$ on the local existence interval $[0, \delta]$ in terms of the parameter $N$.

We recall our aim to give an a-priori bound of $\|\nabla I u(t)\|_{L^{2}}(\mathrm{cf}$. (16)) on any interval $[0, T]$. We want to show this as a consequence of Proposition 2.1 and the estimates for the modified energy just given.

We assume $N \geq 1$ to be a number to be specified later and $s \geq \frac{3}{4}$. Let data $u_{0} \in H^{s}\left(\mathbb{R}^{3}\right)$ be given. Then we have

$$
\begin{aligned}
\left\|\nabla I u_{0}\right\|_{L^{2}}^{2} & \lesssim\left\||\xi| \widehat{u_{0}}(\xi)\right\|_{L^{2}(\{|\xi| \leq N\})}^{2}+\left\|N^{1-s}|\xi|^{s} \widehat{u_{0}}(\xi)\right\|_{L^{2}(\{|\xi| \geq N\})}^{2} \\
& \lesssim\left\|N^{1-s}|\xi|^{s} \widehat{u_{0}}(\xi)\right\|_{L^{2}\left(\mathbb{R}^{3}\right)}^{2}=N^{2(1-s)}\left\|u_{0}\right\|_{\dot{H}^{s}}^{2} \lesssim N^{2(1-s)} .
\end{aligned}
$$

This immediately implies an estimate for $E\left(I u_{0}\right)$. We namely have for $s \geq \frac{3}{4}$ :

$$
\left\|I u_{0}\right\|_{L^{4}\left(\mathbb{R}^{3}\right)}^{4} \lesssim\left\|I u_{0}\right\|_{\dot{H}^{\frac{3}{4}}}^{4} \lesssim\left\|u_{0}\right\|_{H^{s}}^{4}
$$

and trivially $\left\|I u_{0}\right\|_{L^{2}}^{2} \lesssim\left\|u_{0}\right\|_{L^{2}}^{2}$. Thus using the definition of the modified energy and (28):

$$
E\left(I u_{0}\right) \leq c_{0} N^{2(1-s)}
$$

From this we get

$$
\left\|\nabla I u_{0}\right\|_{L^{2}}^{2} \leq c_{0} N^{2(1-s)} .
$$

Our local existence theorem (Proposition 2.1) shows that the Cauchy problem (5), (6) has a unique solution $u$ with $\nabla I u \in X^{0, \frac{1}{2}+}[0, \delta]$ and

$$
\|\nabla I u(\delta)\|_{L^{2}}^{2} \leq\|\nabla I u\|_{X^{0, \frac{1}{2}+}[0, \delta]}^{2} \leq 2\left\|\nabla I u_{0}\right\|_{L^{2}}^{2} \leq 2 c_{0} N^{2(1-s)} .
$$

Here $\delta$ can be chosen subject to the conditions (13), namely (because $s>1 / 2$ ):

$$
\max \left(\delta^{s-\frac{1}{2}-}, \delta^{\frac{s}{2}-} N^{1-s}, \delta^{\frac{1}{2}-} N^{2(1-s)}\right) \sim 1 \Longleftrightarrow \delta \sim \frac{1}{N^{4(1-s)+}} .
$$

In order to reapply the local existence theorem with time steps of equal length we need a uniform bound of $\|\nabla I u(t)\|_{L^{2}}$ at time $t=\delta, t=2 \delta$ etc., which follows from a uniform control over the modified energy. The increment of 
the energy is controlled by (19) and the estimates of this section as follows, provided $s \geq 3 / 4$ :

$$
\begin{aligned}
& \left|E(I u(\delta))-E\left(I u_{0}\right)\right| \\
& \quad \lesssim N^{-1+}\|\nabla I u\|_{X^{0, \frac{1}{2}+}(0, \delta)}^{4}+N^{-1+} \delta^{\frac{1}{4}-}\|\nabla I u\|_{X^{0, \frac{1}{2}+}(0, \delta)}^{3}+N^{-2+}\|\nabla I u\|_{X^{0, \frac{1}{2}+}(0, \delta)}^{6} \\
& \quad+N^{-\frac{5}{2}+}\|\nabla I u\|_{X^{0, \frac{1}{2}+}{ }_{(0, \delta)}^{5}}^{6}+N^{-3+}\|\nabla I u\|_{X^{0, \frac{1}{2}+}(0, \delta)}^{4}+N^{-\frac{5}{2}+} \delta^{\frac{1}{2}}\|\nabla I u\|_{X^{0, \frac{1}{2}+}(0, \delta)}^{3}
\end{aligned}
$$

The last two terms can be neglected in comparison to the others. Thus we get

$$
\begin{aligned}
& \left|E(I u(\delta))-E\left(I u_{0}\right)\right| \\
& \lesssim N^{-1+} N^{4(1-s)}+N^{-1+} N^{-(1-s)+} N^{3(1-s)}+N^{-2+} N^{6(1-s)}+N^{-\frac{5}{2}+} N^{5(1-s)} .
\end{aligned}
$$

One easily checks that the first term is the decisive one, so that

$$
\left|E(I u(\delta))-E\left(I u_{0}\right)\right| \leq c_{1} N^{-1+} N^{4(1-s)},
$$

where $c_{1}=c_{1}\left(c_{0}\right)$. This is easily seen to be bounded by $c_{0} N^{2(1-s)}$ for large $N$. The number of iteration steps to reach a given time $T$ is $\frac{T}{\delta} \sim T N^{4(1-s)+}$. This means that in order to give a uniform bound of the energy of the iterated solutions, namely by $2 c_{0} N^{2(1-s)}$, from the last inequality, the following condition has to be fulfilled:

$$
c_{1} N^{-1+} N^{4(1-s)} T N^{4(1-s)+}<c_{0} N^{2(1-s)},
$$

where $c_{1}=c_{1}\left(2 c_{0}\right)$ (recall here that the initial energy is bounded by $\left.c_{0} N^{2(1-s)}\right)$. This can be fulfilled for $N$ sufficiently large, provided

$$
-1+4(1-s)+4(1-s)<2(1-s) \Longleftrightarrow s>5 / 6 .
$$

This gives the desired bound for $\|\nabla I u(t)\|_{L^{2}}$ on any interval $[0, T]$, i.e. (16) is proved. As explained above this completes the proof of our Theorem 1.1.

\section{References}

[1] Bethuel, F., Saut, J.C.: Travelling waves for the Gross-Pitaevskii equation I. Ann. I. H. Poincaré Phys. Théor. 70, 147-238 (1999)

[2] Bourgain, J.: Scattering in the energy space and below for 3D NLS. J. d'Analyse Math. 75, 267-297 (1998)

[3] Cazenave, T., Haraux, A.: An Introduction to Semilinear Evolution Equations. Oxford science publications, Oxford (1998)

[4] Cazenave, T., Weissler, F.: The Cauchy problem for the nonlinear Schrödinger equation in $H^{s}$. Nonlinear Anal. 14, 807-836 (1990)

[5] Colliander, J., Keel, M., Staffilani, G., Takaoka, H., Tao, T.: Almost conservation laws and global rough solutions to a nonlinear Schrödinger equation. Math. Res. Lett. 9, 659-682 (2002) 
[6] Gallo, C.: The Cauchy problem for defocusing nonlinear Schrödinger equations with non-vanishing initial data at infinity. Comm. Part. Diff. Equa. 33, 729$771(2008)$

[7] Gérard, P.: The Cauchy problem for the Gross-Pitaevskii equation. Ann. I. H. Poincaré Anal. Non-linéaire 23, 765-779 (2006)

[8] Ginibre, J., Tsutsumi, Y., Velo, G.: On the Cauchy problem for the Zakharov system. J. Funct. Anal. 151, 384-436 (1997)

[9] Gross, E.P.: Hydrodynamics of a superfluid condensate. J. Math. Phys. 4, 195$207(1963)$

[10] Grünrock, A.: New applications of the Fourier restriction norm method to wellposedness problems for nonlinear evolution equations. Dissertation University Wuppertal. http://elpub.bib.uni-wuppertal.de/servlets/DocumentServlet? $\mathrm{id}=254(2002)$

[11] Kato, T.: On nonlinear Schrödinger equations II. $H^{s}$-solutions and unconditional well-posedness. J. d'Analyse Math. 67, 281-306 (1995)

[12] Keel, M., Tao, T.: Endpoint Strichartz estimates. Am. J. Math. 120, 955-980 (1998)

[13] Pitaevskii, L.P.: Vortex lines in an imperfect Bose gas. Sov. Phys. JETP 13, 451$454(1961)$

[14] Sulem, C.; Sulem, P.L.: The nonlinear Schrödinger equation. Self-focusing and wave collapse. Appl. Math. Sci. 139, Springer (1999)

Hartmut Pecher

Fachbereich Mathematik und Naturwissenschaften

Bergische Universität Wuppertal

Gaussstr. 20

42097 Wuppertal

Germany

e-mail: pecher@math.uni-wuppertal.de

Received: 17 June 2012.

Accepted: 20 March 2013. 